\title{
Fate of scalar dark matter solitons around supermassive galactic black holes
}

\author{
Philippe Brax and Patrick Valageas $\odot$ \\ Institut de Physique Théorique, Université Paris-Saclay, \\ CEA, CNRS, F-91191 Gif-sur-Yvette Cedex, France \\ Jose A. R. Cembranos(1) \\ Departamento de Física Teórica and Institute of Particle and Cosmos Physics, \\ Universidad Complutense de Madrid, E-28040 Madrid, Spain
}

(Received 10 September 2019; published 24 January 2020)

\begin{abstract}
In scalar-field dark matter scenarios, a scalar-field soliton could form at the center of galactic halos, around the supermassive black holes that sit at the center of galaxies. Focusing on the large scalar-mass limit, where the soliton is formed by the balance between self-gravity and a repulsive self-interaction, we study the infall of the scalar field onto the central Schwarzschild black hole. We derive the scalar-field profile, from the Schwarzschild radius to the large radii dominated by the scalar cloud. We show that the steady state solution selects the maximum allowed flux, with a critical profile that is similar to the transonic solution obtained for the hydrodynamic case. This finite flux, which scales as the inverse of the selfinteraction coupling, is small enough to allow the dark matter soliton to survive for many Hubble times.
\end{abstract}

DOI: 10.1103/PhysRevD.101.023521

\section{INTRODUCTION}

In the past few years, there has been an increasing number of theoretical and experimental analyses investigating the fundamental nature of dark matter (DM). A possible candidate which has attracted a lot of attention is related to ultralight coherent fields. This idea is not new and dates back to the pioneering studies of the QCD axion as a DM candidate [1-3]. More generally, axionlike particles are well motivated by different theories $[4,5]$ with a broad range of masses and couplings [6-15].

The cosmological interest of these DM candidates is associated with the fact that their de Broglie wavelength is of the order of astrophysical scales [16]. This type of coherent DM is constituted of fast oscillating massive scalars $[17,18]$ or other higher-spin bosonic fields [19-22]. For large distances, at both the background and perturbation levels, these coherent fields behave as cold DM (CDM) does [18,23-27]. However, at shorter scales, the matter power spectrum is highly suppressed [25], and the formation of cusps is replaced by DM cores $[28,29]$. These distinctive features of the structure formation associated with light coherent bosonic fields have attracted a lot of attention due to long-standing tensions between standard CDM predictions and different observations on galactic and subgalactic scales [30-37].

In particular, the cored density profiles that this type of DM develops [5,28,38-63] are referred to as solitons. They correspond to stationary or steady solutions of the classical field equations of the bosonic field. In this context, it appears to be necessary to take into account that most galaxies host a supermassive black hole $(\mathrm{BH})$ in their central region [64-66]. The dynamics and phenomenology of ultralight scalar fields within the geometry sourced by a BH have been studied in Refs. [5,54,56,67-74].

In this work, we analyze the impact of anharmonic selfinteractions on scalar DM solitons and their fate in the presence of a central $\mathrm{BH}$. We focus on the quartic case where oscillations of the scalar field are given in terms of elliptic functions that reduce to trigonometric functions in the absence of interactions. These anharmonic corrections introduce large deviations with respect to the standard CDM scenario. They source additional effective pressure (positive for the repulsive case [58,75-80] and negative for the attractive one $[81,82])$, which may alleviate the smallscale problems of CDM [83] and lead to the existence of vortices in galaxies [84]. These modifications can be also used to constrain the parameter space of ultralight coherent DM. In fact, effects on the cosmic microwave background anisotropies [37], large-scale structures [37], and gravitational waves $[85,86]$ have been already considered for this purpose.

Here we focus on the scalar-field profile and behavior around a central $\mathrm{BH}$. We do not address the formation of supermassive BHs within such scalar dark matter scenarios. The existence of these objects is taken as an observational fact, and we assume that it is consistent with scalar-field dark matter scenarios. Actually, for standard CDM (such as weakly interacting massive particles), the formation of supermassive BHs is not straightforward. It does not follow from the DM halo profiles found in cosmological 
simulations, which agree with the well-known NavarroFrenk-White (NFW) profile [87]. In fact, supermassive $\mathrm{BHs}$ are expected to involve baryonic physics, as cooling and dissipation allow baryons to fall into gravitational potential wells. Nonequilibrium physics may also come into play through the mergers of smaller BHs, whereas the initial seeds could result from the remnants of massive stars or the collapse of large gas clouds or of stellar clusters. See, for instance, Ref. [88] for a recent review of scenarios for the assembly of supermassive BHs. Similar baryonic processes should also be present in scalar DM cosmologies; hence, we expect supermassive BHs to form as well in these scenarios.

Thus, in this paper, we investigate the smooth accretion onto the supermassive $\mathrm{BH}$ after a solitonic halo profile has formed on the galactic scale (similar to the NFW halo profile for CDM scenarios). We find that outside the Schwarzschild radius and close enough to the black hole the scalar dynamics are described by a stationary solution with nonvanishing flux. This corresponds to the infall of dark matter into the central BH. Far away from the center, the dynamics reproduce the static soliton behavior, with a solution whose density is nearly constant in the core before falling off rapidly towards zero [89]. This selects a unique solution with constant flux and nearly vanishing velocity far away from the $\mathrm{BH}$, which is similar to the transonic solution obtained for the hydrodynamic case. We find typically that the lifetime of the soliton, despite the falling of matter into the $\mathrm{BH}$, is larger than the age of the Universe. Moreover, the constraints on the density profile of dark matter inferred from the stellar dynamics in the vicinity of the central $\mathrm{BH}[90,91]$ are easily met.

This manuscript is arranged as follows. In Sec. II, we describe the main equations of a generic model of scalar DM within a Schwarzschild geometry, in both isotropic coordinates (Sec. II A 2) and Eddington coordinates (Sec. III D). In Sec. III, we analyze the main features of the scalar DM solitons for the harmonic case. In Sec. IV, we extend this analysis to the self-interacting case determined by a quartic term. In Sec. V, we derive the long lifetime associated with the scalar-field soliton found in the previous section. Finally, the main conclusions are summarized in Sec. VI.

\section{DARK MATTER SCALAR FIELD}

\section{A. Scalar-field action}

The scalar-field action is

$$
S_{\phi}=\int d^{4} x \sqrt{-g}\left[-\frac{1}{2} g^{\mu \nu} \partial_{\mu} \phi \partial_{\nu} \phi-V(\phi)\right] .
$$

We also write the scalar-field potential as

$$
V(\phi)=\frac{m^{2}}{2} \phi^{2}+V_{\mathrm{I}}(\phi),
$$

where $V_{\mathrm{I}}$ is the self-interaction potential. In this work, we focus on the quartic self-interaction potential

$$
V_{\mathrm{I}}(\phi)=\frac{\lambda_{4}}{4} \phi^{4} .
$$

Such scalar fields can play the role of DM and build scalar solitons, i.e., static profiles with a finite core, at the center of galactic halos. These solitons can be the result of the balance between the self-gravity of the scalar cloud and a "quantum pressure" (due to the fact that the underlying equations of motion are the Klein-Gordon equation, or the Schrödinger equation in the nonrelativistic limit, rather than the hydrodynamical Euler equation) or to a repulsive self-interaction, associated with $\lambda_{4}>0$. In this paper, following our previous work [89], we focus on the large scalar-mass limit

$$
m \gg 10^{-21} \mathrm{eV},
$$

which ensures that the quantum pressure is negligible from cosmological to galactic scales. Then, the galactic solitons are due to the balance between gravity and the repulsive self-interaction. In the large scalar-mass limit, the analysis simplifies, and we can derive in the next sections explicit expressions for the scalar-field profile and its inflow onto the supermassive BH. Around a Schwarzschild BH, we shall see below that the large-mass limit becomes defined by the lower bound (40), which is somewhat larger than (4).

\section{B. Schwarzschild metric}

Close to the $\mathrm{BH}$, the contribution from the scalar field is negligible, and the metric is the standard Schwarzschild metric $[92,93]$

$$
d s^{2}=-\left(1-\frac{r_{s}}{\tilde{r}}\right) d t^{2}+\left(1-\frac{r_{s}}{\tilde{r}}\right)^{-1} d \tilde{r}^{2}+\tilde{r}^{2} d \vec{\Omega}^{2},
$$

where $\tilde{r}$ is the Schwarzschild radial coordinate and $r_{s}=$ $2 \mathcal{G M}$ is the Schwarzschild radius of the $\mathrm{BH}$ of mass $M$. Throughout this paper, we work in natural units with $c=1$.

\section{Isotropic coordinates}

We focus on spherically symmetric systems, as we consider a spherical scalar cloud around a supermassive Schwarzschild BH. To simplify the matching with the Newtonian gauge at large scales, we work with the isotropic radial coordinate $r$ and the time $t$ throughout this paper, except in Secs. III D, IV F, and IV G. Then, the static spherically symmetric metric can be written in the isotropic form 


$$
d s^{2}=-f(r) d t^{2}+h(r)\left(d r^{2}+r^{2} d \vec{\Omega}^{2}\right) .
$$

The range of radii from the central $\mathrm{BH}$ can be divided in the following three regimes.

\section{Strong-gravity regime dominated by the $\mathrm{BH}$}

Close to the $\mathrm{BH}$, that is, below a radius $r_{\mathrm{NL}}$, we are in the strong-gravity regime, with nonlinear deviations from the Minkowski metric, dominated by the $\mathrm{BH}$ gravity. There, we recover the Schwarzschild metric but written in the isotropic coordinate system $(t, r)$ of (6) instead of the Schwarzschild coordinate system $(t, \tilde{r})$ of (5). This determines the isotropic metric functions $f(r)$ and $h(r)$ as [93]

$$
\begin{aligned}
\frac{r_{s}}{4}<r<r_{\mathrm{NL}}: f(r) & =\left(\frac{1-r_{s} /(4 r)}{1+r_{s} /(4 r)}\right)^{2}, \\
h(r) & =\left(1+r_{s} /(4 r)\right)^{4},
\end{aligned}
$$

where $r$ is related to the Schwarzschild radial coordinate $\tilde{r}$ by

$$
\tilde{r}>r_{s}, \quad r>\frac{r_{s}}{4}: \quad \tilde{r}=r\left(1+\frac{r_{s}}{4 r}\right)^{2} .
$$

In particular, the $\mathrm{BH}$ event horizon (Schwarzschild radius) reads in these coordinates as

$$
\text { BH horizon: } \tilde{r}=r_{s} \equiv 2 \mathcal{G M}, \quad r=\frac{r_{s}}{4}=\frac{\mathcal{G M}}{2} \text {. }
$$

\section{Weak-gravity regime dominated by the $\mathrm{BH}$}

Further away from the $\mathrm{BH}$ and up to a radius $r_{\mathrm{sg}}$, we are in the weak-gravity regime but still dominated by the supermassive $\mathrm{BH}$ gravity. Therefore, the metric is still given by Eq. (7), where the functions $f(r)$ and $h(r)$ are close to unity. Then, we recover the standard Newtonian gauge

$$
\begin{aligned}
r & \gg r_{\mathrm{NL}}: d s^{2}=-(1+2 \Phi) d t^{2}+(1-2 \Phi) d \vec{r}^{2}, \\
\text { with } \quad \Phi & \ll 1, \quad f=1+2 \Phi, \quad h=1-2 \Phi . \quad
\end{aligned}
$$

From the explicit expressions of $f(r)$ and $h(r)$ in (7), we recover the Newtonian gravitational potential due to the $\mathrm{BH}$ :

$$
r_{\mathrm{NL}} \ll r \ll r_{\mathrm{sg}}: \Phi=-\frac{r_{s}}{2 r}=-\frac{\mathcal{G} M}{r} .
$$

\section{Weak-gravity regime dominated by the scalar cloud}

Beyond the radius $r_{\mathrm{sg}}$, the metric potentials become dominated by the self-gravity of the DM cloud. This also corresponds to the radius where metric fluctuations have decreased down to $10^{-6}-10^{-5}$, as these values are the typical depths of the DM potential wells built on galactic scales. Then, the metric still takes the Newtonian gauge form (10), where $\Phi$ is now given by the scalar-field Poisson equation

$$
r \gg r_{\mathrm{sg}}: \nabla^{2} \Phi=4 \pi \mathcal{G} \rho_{\phi},
$$

where $\rho_{\phi}$ is the scalar-field energy density. This in turn determines the metric functions $f(r)$ and $h(r)$ through the second line in (10).

\section{Equation of motion}

To summarize, at all radii the metric is given by Eq. (6), and in most of this paper we work in this framework. We are dominated by the $\mathrm{BH}$ gravity up to radius $r_{\mathrm{sg}}$. At these small radii, the metric functions $f(r)$ and $h(r)$ are given by Eq. (7). Beyond $r_{\text {sg }}$, we are dominated by the scalar cloud gravity, and the metric functions are determined by the Poisson equation (12). The range dominated by the $\mathrm{BH}$ gravity can be further split over the strong-gravity regime, for $r_{s} / 4<r<r_{\mathrm{NL}}$, and the weak-gravity regime, for $r_{\mathrm{NL}} \ll r \ll r_{\mathrm{sg}}$, where the metric functions can be approximated from (11).

Then, in the static spherical metric (6), the scalar-field Klein-Gordon equation is written

$$
\frac{\partial^{2} \phi}{\partial t^{2}}-\sqrt{\frac{f}{h^{3}}} \vec{\nabla} \cdot(\sqrt{f h} \vec{\nabla} \phi)+f \frac{\partial V}{\partial \phi}=0 .
$$

This also directly follows from the action $S_{\phi}$ written in spherical coordinates:

$$
\begin{aligned}
S_{\phi}= & \int d t d r d \theta d \varphi \sqrt{f h^{3}} r^{2} \sin \theta\left[\frac{1}{2 f}\left(\frac{\partial \phi}{\partial t}\right)^{2}-\frac{1}{2 h}\left(\frac{\partial \phi}{\partial r}\right)^{2}\right. \\
& \left.-\frac{1}{2 h r^{2}}\left(\frac{\partial \phi}{\partial \theta}\right)^{2}-\frac{1}{2 h r^{2} \sin ^{2} \theta}\left(\frac{\partial \phi}{\partial \varphi}\right)^{2}-V(\phi)\right] .
\end{aligned}
$$

\section{FREE SCALAR FIELD}

We first consider the scalar-field inflow profile around the supermassive $\mathrm{BH}$ in the free case, without selfinteractions.

\section{A. Equations of motion}

In the case of the free massive scalar field, that is, when the self-interaction vanishes, the same decomposition of the scalar field as for the nonrelativistic case can be applied. Thus, we can write the real scalar field $\phi$ in terms of a complex scalar field $\psi$ as

$$
\phi=\frac{1}{\sqrt{2 m}}\left(e^{-i m t} \psi+e^{i m t} \psi^{\star}\right) .
$$

As in the nonrelativistic limit, we assume that the time derivative of $\psi$ is much smaller than $m \psi$, that is, 


$$
\dot{\psi} \ll m \psi,
$$

where we note $\dot{\psi}=\partial \psi / \partial t$. Thus, we focus on the large-mass limit. Then, the scalar-field action reads in terms of $\psi$ as

$$
\begin{aligned}
S_{\psi}= & \int d t d r d \theta d \varphi \sqrt{f h^{3}} r^{2} \sin \theta\left[\frac{1}{2 f}\left(i \dot{\psi} \psi^{\star}-i \psi \dot{\psi}^{\star}+m \psi \psi^{\star}\right)\right. \\
& -\frac{1}{2 m h} \frac{\partial \psi}{\partial r} \frac{\partial \psi^{\star}}{\partial r}-\frac{1}{2 m h r^{2}} \frac{\partial \psi}{\partial \theta} \frac{\partial \psi^{\star}}{\partial \theta} \\
& \left.-\frac{1}{2 m h r^{2} \sin ^{2} \theta} \frac{\partial \psi}{\partial \varphi} \frac{\partial \psi^{\star}}{\partial \varphi}-\frac{m}{2} \psi \psi^{\star}\right] .
\end{aligned}
$$

Here we have discarded the fast oscillating terms with factors $e^{ \pm 2 i m t}$, which almost average to zero over a period $2 \pi / m$ because of the slowly evolving assumption (16). The action (17) gives the Euler-Lagrange equation of motion

$$
i \dot{\psi}=-\frac{1}{2 m} \sqrt{\frac{f}{h^{3}}} \vec{\nabla} \cdot(\sqrt{f h} \vec{\nabla} \psi)+m \frac{f-1}{2} \psi .
$$

In the weak-gravity regime (10), for $r \gg r_{s}$, we recover the usual nonrelativistic equation

$$
r \gg r_{s}: i \dot{\psi r}=-\frac{\vec{\nabla}^{2} \psi}{2 m}+m \Phi \psi .
$$

The Madelung transformation [94]

$$
\psi=\sqrt{\frac{\rho}{m}} e^{i s}, \quad \phi=\frac{\sqrt{2 \rho}}{m} \cos (m t-s),
$$

maps the scalar field to an hydrodynamical picture (which breaks where $|\psi|$ vanishes as the phase $s$ becomes ill defined), where $\rho$ plays the role of a density and the phase $s$ defines a curl-free velocity field through

$$
\vec{v}=\frac{\vec{\nabla} s}{m} .
$$

The scalar-field action reads in terms of $\rho$ and $s$ as

$$
\begin{aligned}
S_{\rho, s}= & \int d t d r d \theta d \varphi \sqrt{f h^{3}} r^{2} \sin \theta\left\{-\frac{\rho \dot{s}}{m f}-\frac{1}{2 m^{2} h}\right. \\
& \times\left[\frac{1}{4 \rho}\left(\frac{\partial \rho}{\partial r}\right)^{2}+\rho\left(\frac{\partial s}{\partial r}\right)^{2}\right] \\
& -\frac{1}{2 m^{2} h r^{2}}\left[\frac{1}{4 \rho}\left(\frac{\partial \rho}{\partial \theta}\right)^{2}+\rho\left(\frac{\partial s}{\partial \theta}\right)^{2}\right] \\
& \left.-\frac{1}{2 m^{2} h r^{2} \sin ^{2} \theta}\left[\frac{1}{4 \rho}\left(\frac{\partial \rho}{\partial \varphi}\right)^{2}+\rho\left(\frac{\partial s}{\partial \varphi}\right)^{2}\right]+\frac{\rho}{2 f}-\frac{\rho}{2}\right\} .
\end{aligned}
$$

In the large-mass limit, the density $\rho$ and the velocity $\vec{v}$ remain fixed, while the phase $s$ grows as $m$ from Eq. (21). Thus, formally $\rho$ is of the order of $m^{0}$ and $s$ of the order of $m$. Therefore, in the large-mass limit, the action simplifies to

$$
\begin{aligned}
S_{\rho, s}= & \int d t d r d \theta d \varphi \sqrt{f h^{3}} r^{2} \sin \theta\left\{-\frac{\rho \dot{s}}{m f}-\frac{\rho}{2 m^{2} h}\left(\frac{\partial s}{\partial r}\right)^{2}\right. \\
& -\frac{\rho}{2 m^{2} h r^{2}}\left(\frac{\partial s}{\partial \theta}\right)^{2}-\frac{\rho}{2 m^{2} h r^{2} \sin ^{2} \theta} \rho\left(\frac{\partial s}{\partial \varphi}\right)^{2} \\
& \left.+\frac{\rho(1-f)}{2 f}\right\},
\end{aligned}
$$

where we kept only the leading contributions in $m$. This corresponds to neglecting the quantum pressure term in the Euler equation. This is valid for small spatial density gradients:

$$
|\vec{\nabla} \rho| \ll m \rho .
$$

The Euler-Lagrange equations of motion follow from the derivatives of the action (24) with respect to $s$ :

$$
\dot{\rho}+\sqrt{\frac{f}{h^{3}}} \vec{\nabla} \cdot\left(\sqrt{f h} \rho \frac{\vec{\nabla} s}{m}\right)=0
$$

and with respect to $\rho$ :

$$
\frac{\dot{s}}{m}+\frac{f}{h} \frac{(\vec{\nabla} s)^{2}}{2 m^{2}}=\frac{1-f}{2} .
$$

Taking the gradient of the second equation and substituting the velocity field defined in Eq. (21), we obtain

$$
\begin{gathered}
\dot{\rho}+\sqrt{\frac{f}{h^{3}}} \vec{\nabla} \cdot(\sqrt{f h} \rho \vec{v})=0, \\
\dot{\vec{v}}+\vec{\nabla}\left(\frac{f}{h} \frac{\vec{v}^{2}}{2}\right)=-\frac{\vec{\nabla} f}{2} .
\end{gathered}
$$

In the weak-gravity regime, $r \gg r_{s}$, we recover the usual Newtonian limit of the fluid equations:

$$
\begin{gathered}
r \gg r_{s}: \dot{\rho}+\vec{\nabla} \cdot(\rho \vec{v})=0, \\
\dot{\vec{v}}+(\vec{v} \cdot \vec{\nabla}) \vec{v}=-\vec{\nabla} \Phi .
\end{gathered}
$$

This pressureless Euler equation also corresponds to the motion of free particles in the gravitational potential $\Phi$.

The field $\vec{v}$ can be identified with a standard velocity field only in the nonrelativistic regime, thanks to Eq. (31) that takes the form of the standard hydrodynamical 
Euler equation. In particular, if we expand $s(r)$ around some radius $r_{0}$, we obtain at lowest order $\phi \simeq \phi_{0} \times$ $\cos \left[m t-m v\left(r-r_{0}\right)-s_{0}\right]$. For wave equations, with a propagation speed $c$, we typically have solutions of the form $f(r-c t)$. Comparing with $\cos [m v(t / v-r)]$, we would identify $c=1 / v$, which is clearly incorrect. Thus, $v$ is not related to the phase velocity nor to the group velocity associated to the field $\phi$. In a steady state, the amplitude $\sqrt{2 \rho} / m$ of the oscillations at each radius does not depend on time, in contrast with a wave packet, and $\phi$ resembles more a combination of standing waves $e^{i m t} e^{i k r}$, with the common frequency $m$. We also note that the scalar-field energy density, defined by the energy-momentum tensor as

$$
\rho_{\phi} \equiv-T_{0}^{0}=\frac{1}{2 f}\left(\frac{\partial \phi}{\partial t}\right)^{2}+\frac{1}{2 h}\left(\frac{\partial \phi}{\partial t}\right)^{2}+\frac{m^{2}}{2} \phi^{2},
$$

reads in the large-mass limit as

$$
\rho_{\phi}=\frac{\rho}{f}\left[\sin ^{2}(m t-s)+f \cos ^{2}(m t-s)\right]+\frac{\rho}{h} v^{2} \sin ^{2}(m t-s) .
$$

Averaging over the fast oscillations gives

$$
\left\langle\rho_{\phi}\right\rangle=\rho\left(\frac{f+1}{2 f}+\frac{v^{2}}{2 h}\right) .
$$

In the nonrelativistic limit, we recognize the part associated with the kinetic energy, $\rho v^{2} / 2$, but in the relativistic case the identification of $\rho$ and $v$ is not so clear. In the following, we refer to $\rho$ and $\vec{v}$ as (effective) density and velocity fields, respectively, but one must keep in mind that this identification strictly applies only in the nonrelativistic regime.

\section{B. Steady state}

We can look for stationary solutions of the equations of motion (26) and (27); that is, the density and the velocity fields do not depend on time, but $s$ can have a uniform time dependence. This corresponds to a steady inflow of DM from infinity into the central BH. Restricting to spherically symmetric solutions, the continuity equation (28) gives

$$
\sqrt{f h} r^{2} \rho v_{r}=F,
$$

where $F<0$ is the constant inward flux per unit solid angle, which does not depend on the radius in a steady state. The Euler equation (29) gives

$$
v_{r}=-\sqrt{\frac{h(1-f)}{f}}, \quad \frac{\partial s}{\partial r}=m v_{r},
$$

where we choose the boundary condition $v_{r} \rightarrow 0$ at $r \rightarrow \infty$ to obtain the integration constant. Then, there is no additional uniform time dependence for $s$, we can choose

$$
s(r)=\int^{r} d r m v_{r},
$$

and the complex scalar field $\psi$ is given by Eq. (20). We can check that it satisfies the equation of motion (18) at the leading order in $m$, that is, when we neglect the quantum pressure.

In this large-mass limit of the free scalar field, we recover the infall of independent massive particles, which start at rest at infinity. Their free-falling velocity does not depend on the density, because there are no self-interactions. Then, the density is simply set by Eq. (35), that is, by the requirement of a constant flux

$$
\rho=-\frac{F}{r^{2} h \sqrt{1-f}} .
$$

In particular, the density at the Schwarzschild radius, $r=r_{s} / 4$, is finite, $\rho\left(r_{s} / 4\right)=-F / r_{s}^{2}$, while the velocity $v_{r}$ diverges as $-1 / \sqrt{f} \sim-1 /\left(r-r_{s} / 4\right)$.

We can now check the validity of our large scalarmass limit. The assumption of small time derivative (16) is, of course, satisfied, as $\psi$ does not depend on time. We can see that the density gradient $d \rho / d r$ remains finite down to the Schwarzschild radius. Therefore, the assumption (25) of small density gradients is valid, as long as the Schwarzschild radius is large enough:

$$
r_{s} \gg m^{-1} \text {. }
$$

Using $r_{s}=2 \mathcal{G} M$, this reads as

$$
m \gg 6.7 \times 10^{-19}\left(\frac{M}{10^{8} M_{\odot}}\right)^{-1} \mathrm{eV} .
$$

This lower bound is somewhat larger than the lower bound (4) associated with the growth of cosmological structures. Thus, in this article, we focus on scalar-field masses in the range $10^{-19} \ll m \lesssim 1 \mathrm{eV}$. Our results also apply to the case of an astrophysical BH, with $M \sim 1 M_{\odot}$, if $m \gg 10^{-11} \mathrm{eV}$.

\section{Behavior at the Schwarzschild radius in isotropic coordinates}

In the regime dominated by the $\mathrm{BH}$ gravity, $r \ll r_{\mathrm{sg}}$, we can use the explicit expressions of $f(r)$ and $h(r)$ of Eq. (7). This gives for the density $\rho$ and radial velocity $v_{r}$, respectively,

$$
\rho=-\frac{64 F r^{2}}{\sqrt{r_{s} r}\left(4 r+r_{s}\right)^{3}}, \quad v_{r}=-\sqrt{\frac{r_{s}}{r^{3}} \frac{\left(4 r+r_{s}\right)^{2}}{4\left(4 r-r_{s}\right)}} .
$$

Integrating $v_{r}$ gives the phase $s$ up to an integration constant: 


$$
s=-\frac{m}{2} \sqrt{\frac{r_{s}}{r}}\left[4 r+r_{s}-4 \sqrt{r_{s} r} \ln \left(\frac{2 \sqrt{r / r_{s}}+1}{2 \sqrt{r / r_{s}}-1}\right)\right] .
$$

Expanding around the Schwarzschild radius, we obtain

$$
\begin{aligned}
\rho & =-\frac{F}{r_{s}^{2}}+\frac{3 F\left(4 r-r_{s}\right)^{2}}{8 r_{s}^{4}}+\cdots, \\
v_{r} & =-\frac{8 r_{s}}{4 r-r_{s}}+4+\cdots, \\
s & =-2 m r_{s}\left[1+\ln \left(\frac{4 r-r_{s}}{4 r_{s}}\right)\right]+m\left(4 r-r_{s}\right)+\cdots
\end{aligned}
$$

We recall here that from Eq. (9) the horizon corresponds to $\tilde{r}=r_{s}$ for the Schwarzschild radial coordinate but to $r=$ $r_{s} / 4$ for the isotropic radial coordinate. Thus, the velocity $v_{r}$ and the phase $s$ diverge at the horizon, while the amplitude of the scalar field remains finite. The divergence of the velocity $v_{r}$ at the Schwarzschild radius can seem surprising. Indeed, the velocity of a test particle falling from infinity at rest actually vanishes at the horizon when it is measured by a distant observer. This is due to a strong redshift effect, which makes a distant observer find that the particle takes an infinite amount of time to reach the horizon (whereas nothing strange occurs in the frame of the moving particle as it crosses the horizon). Because the effective velocity field $\vec{v}$ can be identified with a standard velocity only in the nonrelativistic regime via the Euler equation, as discussed below Eq. (31), we do not try to relate explicitly $v_{r}$ or the phase $s$ with test particle velocities in the relativistic regime, close to the horizon. In particular, the divergence of $v_{r}$ does not contradict the vanishing at the horizon of the velocity of a test particle measured by a distant observer.

We shall check in the next section that this divergence is an artificial singularity, due to the choice of the isotropic coordinates, and that the scalar field becomes regular at the horizon by changing to the Eddington time coordinate.

\section{Using the Eddington time coordinate}

\section{Definition of the Eddington time}

The Schwarzschild and isotropic coordinates lead to a coordinate singularity at the Schwarzschild radius $r_{s}$. As is well known, this is not a true geometrical singularity, and one can choose coordinate systems that describe all space down to the physical singularity at $\tilde{r}=0$. We shall check that the scalar field is indeed regular at the horizon when we use the better-behaved coordinate system associated with the Schwarzschild radial coordinate $\tilde{r}$ and the Eddington time $\tilde{t}$, defined by [93]

$$
\tilde{t}=t+r_{s} \ln \left|\frac{\tilde{r}}{r_{s}}-1\right| .
$$

This gives the metric

$$
\begin{aligned}
d s^{2}= & -\left(1-\frac{r_{s}}{\tilde{r}}\right) d \tilde{t}^{2}+2 \frac{r_{s}}{\tilde{r}} d \tilde{t} d \tilde{r}+\left(1+\frac{r_{s}}{\tilde{r}}\right) d \tilde{r}^{2} \\
& +\tilde{r}^{2} d \vec{\Omega}^{2},
\end{aligned}
$$

which is regular over all $\tilde{r}>0$. These coordinates $(\tilde{t}, \tilde{r})$ are directly related to the Eddington-Finkelstein coordinates [93]. Then, we shall check that within the metric (45) the energy-momentum tensor of the scalar field remains finite at the Schwarzschild radius, $\tilde{r}=r_{s}$. In particular, in the coordinates $(\tilde{t}, \tilde{r})$ and for spherically symmetric configurations, the density defined by the time-time component of the energy-momentum tensor reads

$$
\tilde{\rho}_{\phi} \equiv-\tilde{T}_{0}^{0}=\frac{2-f}{2}\left(\frac{\partial \phi}{\partial \tilde{t}}\right)^{2}+\frac{f}{2}\left(\frac{\partial \phi}{\partial \tilde{r}}\right)^{2}+V,
$$

and the partial derivatives are related by

$$
\frac{\partial \phi}{\partial \tilde{t}}=\frac{\partial \phi}{\partial t}, \quad \frac{\partial \phi}{\partial \tilde{r}}=\frac{\partial \phi}{\partial r} \frac{1}{\sqrt{f h}}+\frac{\partial \phi}{\partial t}\left(1-\frac{1}{f}\right) .
$$

\section{Behavior at the Schwarzschild radius in Eddington coordinates}

Substituting the results (43) into the Madelung decomposition (20) and using the Eddington time (44) with the Schwarzschild radial coordinate, as in the metric (45), we obtain at leading order for $\tilde{r} \rightarrow r_{s}$

$$
\phi=\sqrt{\frac{-2 F}{m^{2} r_{s}^{2}}} \cos \left[m\left(\tilde{t}+\tilde{r}+r_{s}(1-\ln 4)\right)\right]+\cdots .
$$

Thus, the scalar field is well defined at the horizon, provided we use regular coordinates, and as expected we recover a fully ingoing solution. The divergence of the "velocity" $v_{r}$ and the phase $s$ at the horizon in the Schwarzschild and isotropic metrics is due to the fact that the time $t$ is not an appropriate coordinate at the horizon. As noticed above, for the infall of test particles, this shortcoming of the Schwarzschild and isotropic metrics leads to the fact that a distant observer never sees the particles cross the horizon. For the scalar field, it gives rise instead to a divergence of $v_{r}$ and $s$. This divergence of the phase $s(r)$ in isotropic coordinates precisely combines with the exponential factor $e^{-i m t}$ in Eq. (15) so as to give a regular expression in terms of $(\tilde{t}, \tilde{r})$, once we use an appropriate time coordinate.

In a similar fashion, the energy-momentum tensor associated with the Schwarzschild or isotropic metrics diverges at the Schwarzschild radius, but the one associated with the Eddington metric (45) remains finite. 


\section{Density profile}

From Eqs. (20) and (46), the energy density associated with the Eddington coordinates is given, at leading order in the large- $m$ limit, by

$$
\begin{aligned}
\tilde{\rho}_{\phi}= & \rho\left\{\sin ^{2}(m t-s)\left[2-f+\frac{1}{f}(1-f-\sqrt{1-f})^{2}\right]\right. \\
& \left.+\cos ^{2}(m t-s)\right\} .
\end{aligned}
$$

In terms of the flux $F$, we obtain using Eq. (38)

$$
\left\langle\tilde{\rho}_{\phi}\right\rangle=-\frac{F}{r_{s}^{2}} \frac{r_{s}^{2}}{2 r^{2} h \sqrt{1-f}}\left[3-f+\frac{1}{f}(1-f-\sqrt{1-f})^{2}\right],
$$

where we took the average over the fast oscillations of period $2 \pi / m$. As expected, this scalar-field energy density remains finite at the Schwarzschild radius, with

$$
\tilde{r}=r_{s}, \quad r=\frac{r_{s}}{4}: \quad\left\langle\tilde{\rho}_{\phi}\right\rangle=-\frac{3 F}{2 r_{s}^{2}} .
$$

At larger radii that are still dominated by the $\mathrm{BH}$ gravitational potential, this gives

$$
r_{s} \ll r \ll r_{\mathrm{sg}}:\left\langle\tilde{\rho}_{\phi}\right\rangle \propto r^{-3 / 2} \quad \text { and } \quad v_{r} \propto r^{-1 / 2}
$$

The scaling $v_{r} \propto r^{-1 / 2}$ corresponds to the free fall from rest at infinity, which also gives $v_{r}^{2} \sim \Phi \sim \mathcal{G} M / r$. The requirement of constant flux through spherical shells then implies $\rho_{\phi} \propto r^{-3 / 2}$. The density $\rho_{\phi}$ grows linearly with $|F|$, as there are no self-interactions (and we neglect self-gravity near the $\mathrm{BH}$ ).

The unit velocity obtained in the ingoing wave (48), or of the order of unity in Eq. (51) if we define an effective velocity by $F=\left\langle\tilde{\rho}_{\phi}\right\rangle r^{2} \tilde{v}_{r}^{\text {eff }}$, shows that as expected the scalar-field dynamics are strongly relativistic at the Schwarzschild radius. In particular, the phase $s$ is not small, and the exponent $e^{i s}$ of the wave function $\psi$ cannot be expanded over, as it must precisely combine with the factor $e^{-i m t}$ to give the regular solution (48). Also, whereas $\rho$ given by Eq. (41) remains finite at the Schwarzschild radius, $s$ given by Eq. (42) diverges. This means that, whereas density gradients remain small, as compared with the scalar mass, as long as the bound (39) is fulfilled, the radial derivatives of the phase $s$ and of the wave functions $\psi$ and $\phi$ are not small and even diverge at the Schwarzschild radius. Again, this means that one cannot use a perturbative approach in the scalar field, even in the large scalar-mass limit. One must keep the nonlinearities of the scalarfield phase.

\section{QUARTIC INTERACTION}

We now consider the scalar-field inflow profile around the supermassive $\mathrm{BH}$ in the case of quartic self-interactions (3).

\section{A. Large-mass approximation}

For spherical modes and the quartic self-interaction (3), the nonlinear Klein-Gordon equation (13) reads

$\frac{\partial^{2} \phi}{\partial t^{2}}-\sqrt{\frac{f}{h^{3}}} \frac{1}{r^{2}} \frac{\partial}{\partial r}\left[\sqrt{f h} r^{2} \frac{\partial \phi}{\partial r}\right]+f m^{2} \phi+f \lambda_{4} \phi^{3}=0$.

If we discard the radial derivatives, we recognize the standard Duffing equation, which describes a nonlinear oscillator with a cubic nonlinearity [95]. Its solution can be written as $\phi_{0} \operatorname{cn}(\omega t-\beta, k)$, where $\operatorname{cn}(u, k)$ is the Jacobi elliptic function $[96,97]$ of argument $u$ and modulus $k$. The angular frequency $\omega$ and the modulus $k$ are functions of the amplitude $\phi_{0}$, as for anharmonic oscillators the frequency depends on the amplitude of the oscillations. The harmonic case $\lambda_{4}=0$ corresponds to $k=0$ as $\operatorname{cn}(u, 0)=\cos (u)$. For general $k$, the Jacobi elliptic function $\operatorname{cn}(u, k)$ is a periodic function of $u$ with period $4 \mathbf{K}$, where $\mathbf{K}(k)$ is the complete elliptic integral of the first kind, defined by $[96,97]$

$$
0 \leq k<1: \mathbf{K}(k)=\int_{0}^{\pi / 2} \frac{d \theta}{\sqrt{1-k^{2} \sin ^{2} \theta}},
$$

and $\mathbf{K}(0)=\pi / 2$.

Taking into account the radial dependence, we can look for a solution of the form

$$
\phi=\phi_{0}(r) \operatorname{cn}[\omega(r) t-\mathbf{K}(r) \beta(r), k(r)],
$$

where we noted $\mathbf{K}(r) \equiv \mathbf{K}[k(r)]$. This is understood as the leading-order approximation in the limit $m \rightarrow \infty$, where spatial gradients of the amplitude $\phi_{0}$ and the modulus $k$ are much below $m$, while both $\omega$ and $\beta$ are of the order of $m$. The amplitude $\phi_{0}$, the angular frequency $\omega$, the phase $\beta$, and the modulus $k$ are slow functions of the radius. Thus, Eq. (55) is a generalization of the free-scalar solution (20) to the case of nonzero quartic self-interaction, in the same large-mass approximation.

We could absorb the factor $\mathbf{K}(r)$ in Eq. (55) in $\beta(r)$ and write the solution as $\phi_{0} \operatorname{cn}(\omega t-\beta, k)$. However, it is convenient to introduce the factor $\mathbf{K}$ in the definition of $\beta$ to simplify the Fourier expansion (57) below, which also simplifies the radial derivative (59) below. Removing the factor $\mathbf{K}$ in Eq. (55) would make new factors $\mathbf{K}$ and $\mathbf{K}^{\prime}$ appear in Eqs. (57) and (59) below.

The factors $\phi_{0}, \omega, \beta$, and $k$ are then determined by the equation of motion (53). This will relate them to the 
scalar-field physical parameters $m$ and $\lambda_{4}$, and to the mass of the BH, through Eqs. (62) and (63) below.

First, to ensure that spatial gradients do not increase with time, we note that the field at each radius must oscillate in phase. Otherwise, the phase difference between the fields at two arbitrarily close radii would eventually become large, giving rise to a secular growth of radial gradients. Denoting the common period of the oscillations by $T=2 \pi / \omega_{0}$, where $\omega_{0}$ is the common angular frequency, we can see from Eq. (55) that we must have at each radius $\omega(r) T=4 \mathbf{K}(r)$, because the period of the Jacobi function of modulus $k$ is $4 \mathbf{K}$. Therefore, the function $\omega(r)$ is set by the modulus $k(r)$ according to

$$
\omega(r)=\frac{2 \mathbf{K}(r)}{\pi} \omega_{0}
$$

This synchronous oscillation can also be seen from the Fourier series expansion of the Jacobi elliptic function $[96,97]$. Substituting into Eq. (55), we obtain

$\phi=\phi_{0} \frac{2 \pi}{k \mathbf{K}} \sum_{n=0}^{\infty} \frac{q^{n+1 / 2}}{1+q^{2 n+1}} \cos \left[(2 n+1)\left(\omega_{0} t-\pi \beta / 2\right)\right]$,

with $q=e^{-\pi \mathbf{K}^{\prime} / \mathbf{K}}$, where $\mathbf{K}^{\prime}=\mathbf{K}\left(k^{\prime}\right)$ with $k^{\prime}=\sqrt{1-k^{2}}$. This expression explicitly shows the global oscillation of the field, with the common fundamental angular frequency $\omega_{0}$.

From Eq. (55), the time derivative is

$$
\frac{\partial \phi}{\partial t}=\phi_{0} \omega \frac{\partial \mathrm{cn}}{\partial u}
$$

At leading order in the large- $m$ limit, the radial derivative reads from Eq. (57) as

$$
\frac{\partial \phi}{\partial r}=-\phi_{0} \mathbf{K} \beta^{\prime} \frac{\partial \mathrm{cn}}{\partial u}+\cdots
$$

where the dots stand for subleading terms, as we assume that the phase $\beta$ is formally of the order of $m$. Here, $\beta^{\prime}=d \beta / d r$. Substituting into the nonlinear Klein-Gordon equation (53) gives

$\phi_{0}\left[\omega^{2}-\frac{f}{h}\left(\mathbf{K} \beta^{\prime}\right)^{2}\right] \frac{\partial^{2} \mathrm{cn}}{\partial u^{2}}+f m^{2} \phi_{0} \mathrm{cn}+f \lambda_{4} \phi_{0}^{3} \mathrm{cn}^{3}=0$,

where we kept only the term of the order of $m^{2}$ among the radial derivative contributions. Thus, at this order, we can see that the radial derivatives do not change the structure of Eq. (60). This is why it again admits the Jacobi elliptic function as a solution. Thus, using the property

$$
\frac{\partial^{2} \mathrm{cn}}{\partial u^{2}}=\left(2 k^{2}-1\right) \mathrm{cn}-2 k^{2} \mathrm{cn}^{3},
$$

the Klein-Gordon equation (60) is satisfied as soon as the coefficients of the factors $\mathrm{cn}$ and $\mathrm{cn}^{3}$ vanish. This gives the two conditions

$$
\begin{gathered}
\frac{\pi^{2} f}{4 h} \beta^{\prime 2}=\omega_{0}^{2}-\frac{f m^{2} \pi^{2}}{\left(1-2 k^{2}\right) 4 \mathbf{K}^{2}}, \\
\frac{\lambda_{4} \phi_{0}^{2}}{m^{2}}=\frac{2 k^{2}}{1-2 k^{2}} .
\end{gathered}
$$

We recover in Eq. (63) that the free scalar case $\lambda_{4}=0$ corresponds to $k=0$. Equation (62) is the generalization of the Euler equation (27), $\pi \beta^{\prime} /(2 m)$ plays the role of the radial velocity $v_{r}=m^{-1} d s / d r$, and $\pi \beta / 2$ plays the role of the phase $s$. More precisely, Eq. (62) can be rewritten as a relativistic dispersion relation for a particle of mass $m$, i.e., $E^{2}=p_{r}^{2}+m^{2}$, where we identify $p_{r}=\sqrt{\frac{1-2 k^{2}}{h} \mathbf{K}} \beta^{\prime}$ and $E=\sqrt{\frac{1-2 k^{2}}{f}} \frac{2 \mathbf{K}}{\pi} \omega_{0}$ in the local Minkowski frame. In the large-radius limit, as we shall see in Sec. IV B 1 b corresponding to $k \ll 1$, this reduces to the dispersion relation of the nonrelativistic particles as identified thanks to the Euler equation, and we have $p_{r} \simeq \pi \beta^{\prime} / 2$. In the general case, we can identify the velocity field as $v_{r} \equiv \frac{p_{r}}{E}$. In the relativistic regime close to the $\mathrm{BH}$, this velocity goes to unity. However, the connection between the dispersion relation and a velocity field is mostly formal, as beyond the nonrelativistic regime there is no direct link between the profile of the solution $\phi$ given by Eq. (55) and a particle interpretation.

\section{B. Boundary conditions}

\section{Large-radius boundary condition}

At large radii, $r \gg r_{\mathrm{sg}}$, the gravitational field is small and set by the self-gravity of the scalar cloud. Therefore, we match the solution (55) to the soliton profile obtained for the self-gravitational nonrelativistic scalar cloud [89].

Scalar-field soliton.-In this regime, we can decompose the scalar field $\phi$ as in Eq. (15) and use the Madelung transformation (20) for the complex field $\psi$. Taking into account the quartic self-interaction, which is subdominant with respect to the quadratic potential $m^{2} \phi^{2} / 2$, the continuity equations (26) and (28) take again the usual form (30)

$$
\dot{\rho}+\frac{\vec{\nabla} \cdot(\rho \vec{\nabla} s)}{m}=0, \quad \dot{\rho}+\vec{\nabla} \cdot(\rho \vec{v})=0,
$$

whereas the Euler equations (27) and (29) become 


$$
\begin{aligned}
\frac{\dot{s}}{m}+\frac{(\vec{\nabla} s)^{2}}{2 m^{2}} & =-\left(\Phi+\Phi_{\mathrm{I}}\right), \\
\dot{\vec{v}}+(\vec{v} \cdot \vec{\nabla}) \vec{v} & =-\vec{\nabla}\left(\Phi+\Phi_{\mathrm{I}}\right),
\end{aligned}
$$

where $\Phi_{\mathrm{I}}$ is given by [89]

$$
\Phi_{\mathrm{I}}(\rho)=\frac{\rho}{\rho_{a}}, \quad \rho_{a} \equiv \frac{4 m^{4}}{3 \lambda_{4}} .
$$

This pressure associated with the self-interaction $\Phi_{\text {I }}$ allows the scalar cloud to reach an hydrostatic equilibrium, where this repulsive self-interaction balances the self-gravity. This gives the soliton profile [89]

$\rho(r)=\rho_{s}(0) \frac{\sin \left(r / r_{a}\right)}{r / r_{a}}, \quad \Phi_{\mathrm{I}}(r)=\Phi_{\mathrm{I} s}(0) \frac{\sin \left(r / r_{a}\right)}{r / r_{a}}$,

with $\vec{v}=0$ and

$$
r_{a}=\frac{1}{\sqrt{4 \pi \mathcal{G} \rho_{a}}} .
$$

The soliton has a flat inner core and a finite radius $R_{s}=\pi r_{a}$. Inside the soliton, the hydrostatic equilibrium condition (65) gives $\vec{\nabla}\left(\Phi+\Phi_{\mathrm{I}}\right)=0$, and we have

$$
r \leq R_{s}: \Phi+\Phi_{\mathrm{I}}=\alpha,
$$

where $\alpha$ is a constant, given by the value of the Newtonian potential at the boundary of the soliton,

$$
\alpha=\Phi\left(R_{s}\right),
$$

as $\Phi_{\mathrm{I}}\left(R_{s}\right)=0$. In terms of the scalar fields $\psi$ and $\phi$, this gives

$$
\psi=\sqrt{\frac{\rho}{m}} e^{-i \alpha m t}, \quad \text { hence, } \quad s=-\alpha m t,
$$

and

$$
\phi=\frac{\sqrt{2 \rho}}{m} \cos [(1+\alpha) m t]
$$

Large-radius solution.-At large radii but within the soliton radius, $r_{\mathrm{sg}} \ll r \ll R_{s}$, we are in the weak-gravity regime and we approach the soliton core solution, with $\Phi \simeq \Phi_{s}(0) \lesssim 10^{-5}$ and $\rho \simeq \rho_{s}(0)$. We also have $\Phi_{\mathrm{I}}=\alpha-$ $\Phi \simeq-\Phi_{s}(0)$, and the self-interaction potential $V_{\mathrm{I}} \sim \rho \Phi_{\mathrm{I}} \ll$ $\rho$ is much smaller than the quadratic part; hence, $\lambda_{4} \phi^{4} \ll m^{2} \phi^{2}$. Therefore, we can see from Eq. (63) that we have at leading order

$$
k^{2}=\frac{\lambda_{4} \phi_{0}^{2}}{2 m^{2}}+\cdots \ll 1,
$$

where the dots stand for higher-order terms. From the expansion (57) and the series expansions [97]

$$
\begin{aligned}
& \mathbf{K}(k)=\frac{\pi}{2}\left(1+\frac{k^{2}}{4}+\cdots\right), \\
& q(k)=\frac{k^{2}}{16}\left(1+\frac{k^{2}}{8}+\cdots\right),
\end{aligned}
$$

we obtain at leading order

$$
k \ll 1: \phi=\phi_{0} \cos \left(\omega_{0} t-\pi \beta / 2\right)+\cdots .
$$

The comparison with Eq. (72) gives

$$
r_{\mathrm{sg}} \ll r \ll R_{s}: \phi_{0}(r)=\frac{\sqrt{2 \rho_{s}(0)}}{m}, \quad \beta \simeq 0,
$$

and

$$
\omega_{0}=(1+\alpha) m
$$

Indeed, as the soliton solution (72) corresponds to hydrostatic equilibrium with $\vec{v}=0$, the velocity $\beta$ must become negligible at large radii in order to match with the soliton. We can now check that this is consistent with Eqs. (62) and (63). Equation (62) with $\beta=0$ gives, at leading order in $\Phi$ and $k^{2}$,

$$
\omega_{0}=m\left(1+\Phi+\frac{3}{4} k^{2}\right) .
$$

On the other hand, Eq. (63) gave Eq. (73). Using Eq. (77), this yields

$$
k^{2}=\frac{\lambda_{4} \rho}{m^{4}}=\frac{4}{3} \Phi_{\mathrm{I}} .
$$

Then, Eq. (79) reads $\omega_{0}=m\left(1+\Phi+\Phi_{\mathrm{I}}\right)=m(1+\alpha)$, where we used the hydrostatic result (69), and we recover Eq. (78). This shows that this large-radius asymptote is selfconsistent, provided $\beta$ is negligible. This gives the largeradius asymptotic values of $\phi_{0}(r)$ and $k(r)$, from Eqs. (77) and (80), in the constant-density core of the soliton. The uniform oscillation frequency $\omega_{0}$ is then set by this largeradius boundary condition in Eq. (78). Note that typically $\alpha \lesssim 10^{-5}$ from Eq. (70). Thus, the angular oscillation frequency $\omega_{0}$ remains very close to $m$.

\section{Small-radius boundary condition}

Close to the Schwarzschild radius, we can expect the self-interaction pressure to be negligible and to recover the free-scalar infall (36) (but we shall see below that the selfinteraction plays a role for the scalar profile down to the Schwarzschild radius, as it dictates the constant flux of the steady state). Indeed, as long as $k$ remains below $1 / \sqrt{2}$, 
the last term in the generalized Euler equation (62) becomes negligible as $f \rightarrow 0$ at the Schwarzschild radius, and Eq. (62) gives

$$
r \rightarrow \frac{r_{s}}{4}: \frac{\pi}{2} \beta^{\prime}=-\omega_{0} \sqrt{\frac{h}{f}} .
$$

This agrees indeed with Eq. (36) (except for the prefactor $\alpha$ associated with the finite soliton size).

\section{Steady state and constant flux}

So far, any profile $k(r)$ with the outer boundary condition (80) and $k(r)<1 / \sqrt{2}$ at all radii provides a leadingorder solution (55). Indeed, given $k(r)$, Eq. (62) provides the velocity $\beta^{\prime}$, while Eq. (63) provides the amplitude $\phi_{0}$, i.e., the "density." Clearly, we do not expect such a large space of physical solutions. It would seem more natural to recover a specific profile, such as the unique transonic solution found for hydrodynamics in nonrelativistic [98] and relativistic [99] infall. In fact, at this stage we miss a constant flux constraint associated with a continuity equation, as in Eq. (35). In the relativistic case, the continuity equation is associated with the component $\nu=0$ of the conservation equations $\nabla_{\mu} T_{\nu}^{\mu}=0$. The energy-momentum tensor of the scalar field $\phi$ gives

$$
\rho_{\phi} \equiv-T_{0}^{0}=\frac{1}{2 f}\left(\frac{\partial \phi}{\partial t}\right)^{2}+\frac{1}{2 h}\left(\frac{\partial \phi}{\partial r}\right)^{2}+V
$$

and

$$
T_{0}^{r}=\frac{1}{h} \frac{\partial \phi}{\partial r} \frac{\partial \phi}{\partial t} .
$$

At leading order in the large-mass limit, we obtain from Eqs. (55), (58), (59), (62), and (63)

$$
\begin{aligned}
\rho_{\phi}= & \frac{\left(1-k^{2}\right) m^{2} \phi_{0}^{2}}{2\left(1-2 k^{2}\right)}+\phi_{0}^{2} \frac{\left(\mathbf{K} \beta^{\prime}\right)^{2}}{h} \\
& \times\left[1-k^{2}+\left(2 k^{2}-1\right) \mathrm{cn}^{2}-k^{2} \mathrm{cn}^{4}\right]
\end{aligned}
$$

and

$$
T_{0}^{r}=-\phi_{0}^{2} \omega \frac{\mathbf{K} \beta^{\prime}}{h}\left(\frac{\partial \mathrm{cn}}{\partial u}\right)^{2}
$$

Then, using again Eqs. (58), (59), and (61), we can check that the conservation equation $\nabla_{\mu} T_{0}^{\mu}=0$, which reads

$$
\dot{\rho}-\frac{1}{\sqrt{f h^{3}} r^{2}} \frac{\partial}{\partial r}\left[\sqrt{f h^{3}} r^{2} T_{0}^{r}\right]=0
$$

is satisfied at the leading order. We can note that $\rho_{\phi}$ is not constant with time, as the terms $\mathrm{cn}^{2}$ and $\mathrm{cn}^{4}$ in the bracket in Eq. (84) oscillate with the frequency $\omega_{0}$. At the leading order, the continuity equation (86) is governed by the fast oscillation of these terms. However, to ensure that subleading orders do not show secular terms that grow with time, we clearly require that in the steady state the averaged value of $\rho_{\phi}$ over one oscillation period does not depend on time. This gives the condition of constant flux

$F=-\sqrt{f h^{3}} r^{2}\left\langle T_{0}^{r}\right\rangle=\sqrt{f h} r^{2} \phi_{0}^{2} \omega \mathbf{K} \beta^{\prime}\left\langle\left(\frac{\partial \mathrm{cn}}{\partial u}\right)^{2}\right\rangle$,

where $\langle\ldots\rangle$ denotes the average over one oscillation period $T=2 \pi / \omega_{0}$. Using Eqs. (56), (62), (63), and (78), we can write the flux in terms of $k(r)$ :

$$
\begin{aligned}
F= & F_{s} x^{2} h\left(\frac{2 \mathbf{K}}{\pi}\right)^{2}\left\langle\left(\frac{\partial \mathrm{cn}}{\partial u}\right)^{2}\right\rangle \frac{2 k^{2}}{1-2 k^{2}} \\
& \times \sqrt{1-\frac{\pi^{2} f}{(1+\alpha)^{2} 4 \mathbf{K}^{2}\left(1-2 k^{2}\right)}},
\end{aligned}
$$

where we defined the dimensionless radial coordinate

$$
x=\frac{r}{r_{s}}>\frac{1}{4}
$$

and the characteristic flux

$$
F_{s}=-\frac{r_{s}^{2} m^{4}(1+\alpha)^{2}}{\lambda_{4}} \simeq-\frac{r_{s}^{2} m^{4}}{\lambda_{4}},
$$

as typically $\alpha \lesssim 10^{-5}$. The average value of $\left(\frac{\partial \mathrm{cn}}{\partial u}\right)^{2}$ is

$$
\left\langle\left(\frac{\partial \mathrm{cn}}{\partial u}\right)^{2}\right\rangle=1-k^{2}+\left(2 k^{2}-1\right) C_{2}-k^{2} C_{4}
$$

with [95]

$$
\begin{gathered}
C_{2} \equiv\left\langle\mathrm{cn}^{2}\right\rangle=\frac{1}{k^{2}}\left(\frac{\mathbf{E}}{\mathbf{K}}+k^{2}-1\right), \\
C_{4} \equiv\left\langle\mathrm{cn}^{4}\right\rangle=\frac{1}{3 k^{2}}\left(2\left(2 k^{2}-1\right) C_{2}+1-k^{2}\right),
\end{gathered}
$$

where $\mathbf{E}(k)$ is the complete elliptic integral of the second kind, defined by $[96,97]$

$$
0 \leq k<1: \mathbf{E}(k)=\int_{0}^{\pi / 2} d \theta \sqrt{1-k^{2} \sin ^{2} \theta} .
$$

We can see from Eq. (90) that the flux diverges as $1 / \lambda_{4}$. This is not surprising, since for a vanishing self-interaction we must recover the free-scalar case studied in Sec. III, where the flux is arbitrary and has no upper bound. We also find that the flux scales as $r_{s}^{2} m^{4}$, which is also natural, as we 


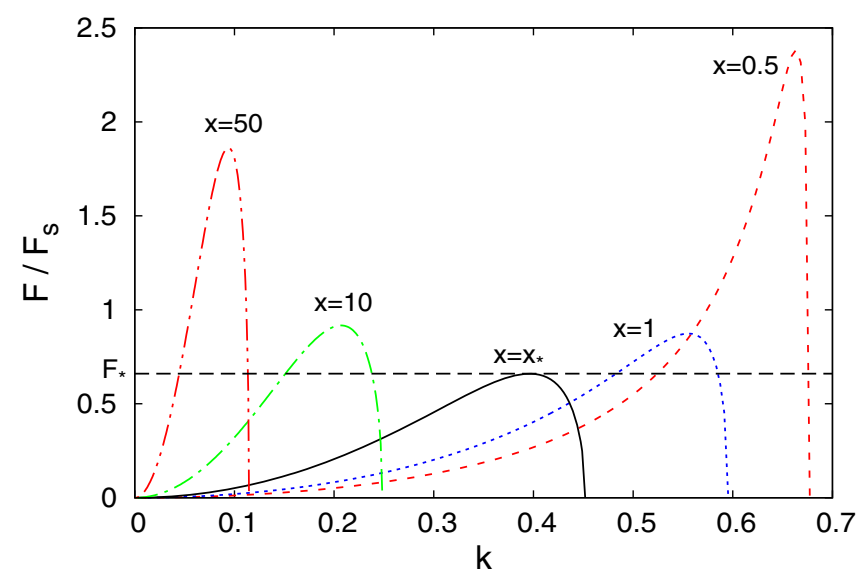

FIG. 1. Normalized flux $F(k, x) / F_{s}$ as a function of the modulus $k$, for various values of the radial coordinate $x$, from Eq. (88). The horizontal dotted line is the minimum value $F_{\star} \simeq$ 0.66 of the peak, reached for $x=x_{\star} \simeq 2.43$.

can expect $F \sim \rho r^{2} v_{r}$, with $r=r_{s} / 4, \quad v_{r} \sim 1$ at the Schwarzschild radius, and $\rho \sim m^{4}$ from dimensional analysis.

\section{Critical solution}

\section{Function $\boldsymbol{F}(\boldsymbol{k}, \boldsymbol{x})$}

For each radius $x$, Eq. (88) gives the flux $F$ as a function of $k$. We show in Fig. 1 the normalized flux $F / F_{s}$ as a function of the modulus $k$ for several values of the radial coordinate $x$. The modulus $k$ is constrained to range between 0 and the value $k_{+}(x)<1 / \sqrt{2}$ where the square root vanishes. The flux vanishes at both boundaries, $k=0$ and $k=k_{+}$, and shows a single maximum $\left|F_{\max }(x)\right|$ at a position $k_{\max }(x)$ somewhat below $k_{+}(x)$. The upper bound $k_{+}$and the peak at $k_{\max }$ shift to lower values as $x$ grows. The maximum $\left|F_{\max }(x)\right|$ grows at both small and large $x$ and shows a minimum at $x_{\star} \simeq 2.43$ with

$$
F_{c} \equiv F_{\max }\left(x_{\star}\right)=F_{\star} F_{s} \quad \text { with } \quad F_{\star} \simeq 0.66 .
$$

We show $F_{\max }(x) / F_{s}$ in Fig. 2. In Figs. 1 and 2, we use for the metric functions $h(x)$ and $f(x)$ the Schwarzschild functions (7). At the transition radius $r_{\mathrm{sg}}$, the gravitational potential receives equal contributions from the central $\mathrm{BH}$ and the scalar cloud, and at a larger radius inside the soliton core it remains almost constant, equal to the soliton core value $\Phi_{s}(0)$. Therefore, beyond $r_{\text {sg }}$ the factors $h$ and $f$ are almost constant, and the flux function $F(x, k)$ keeps a constant shape in $k$, with a simple multiplicative factor $x^{2}$. Thus, beyond $r_{\mathrm{sg}}$ the peak value $\left|F_{\max }(x)\right|$ keeps increasing as $x^{2}$.

This behavior of $F(k, x)$ selects a unique value for the flux, in a fashion similar to the unique transonic solution found in the case of hydrodynamical infall onto a $\mathrm{BH}$

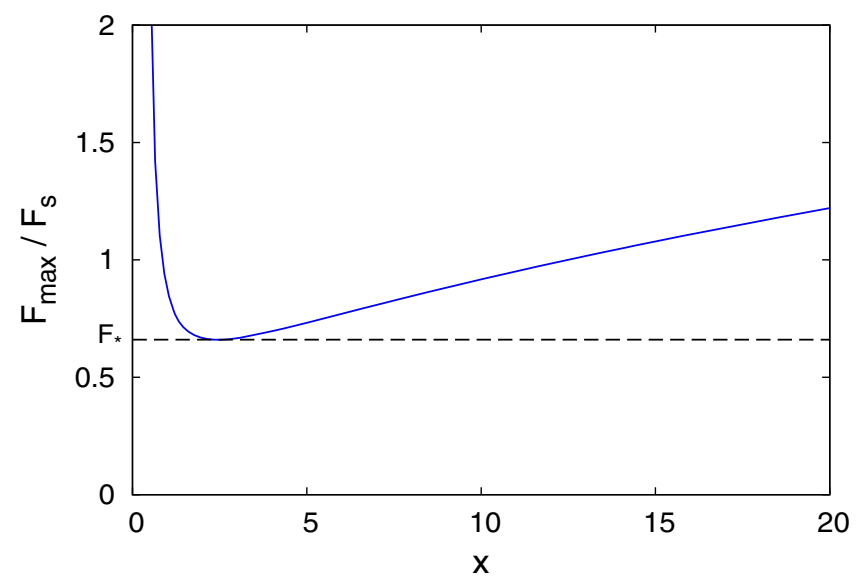

FIG. 2. Peak value $F_{\max }(x) / F_{s}$ as a function of the radial coordinate $x$. The horizontal dotted line is the minimum value $F_{\star} \simeq 0.66$.

$[98,99]$. First, we can see that $|F|$ must be smaller than or equal to the critical value $\left|F_{c}\right|$; otherwise, there would exist no solution $k(x)$ to the flux constraint equation (88) around $x_{\star}$. If $|F|<\left|F_{c}\right|$, there exist two distinct solutions $k_{1}(x)<$ $k_{2}(x)$ at each radius, on either side of the peak $k_{\max }(x)$, and a continuous function $k(x)$ must remain on the same side of the peak throughout. It is only for the critical value $F=F_{c}$ that the function $k(x)$ can switch from the branch $k_{1}(x)$ to $k_{2}(x)$, at the radius $x_{\star}$ where both solutions coincide with the peak. The two solutions $k_{1}(x)<k_{2}(x)$ are shown in Fig. 3 for $F=F_{c} / 3$ (the upper and lower dashed curves that do not meet) and for $F=F_{c}$ [the inner dotted curves that meet at $x_{\star} \simeq 2.43$, which coincide with the critical solution $k_{c}(x)$, shown by the solid line, on either side of $x_{\star}$ ].

As we shall see below, the boundary conditions require that $k=k_{2}(x)$ at large radii and $k=k_{1}(x)$ close to the Schwarzschild radius. Therefore, the function $k(x)$ must

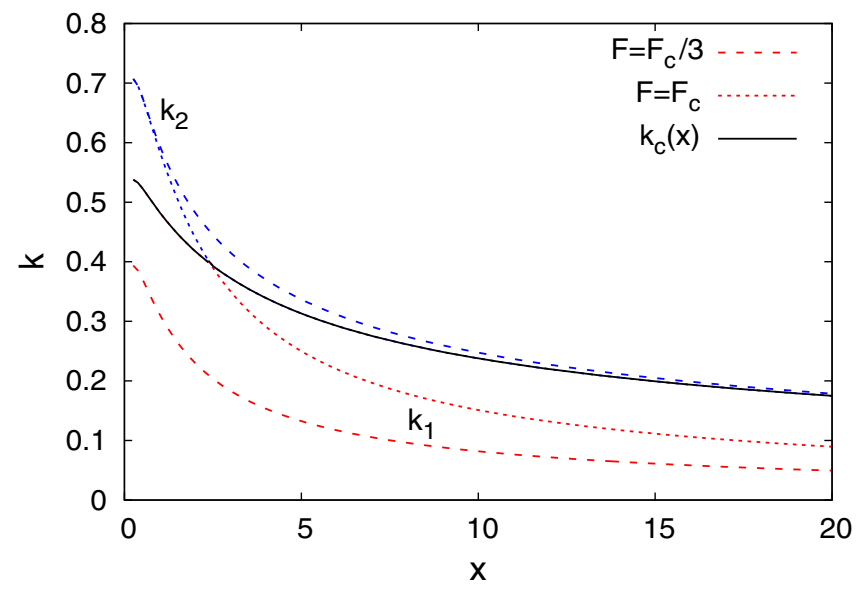

FIG. 3. Moduli $k_{1}(x)$ and $k_{2}(x)$ for a constant flux $F_{c} / 3$ (dashed lines) and $F_{c}$ (dotted lines). The critical modulus $k_{c}(x)$ (solid line) is equal to $k_{1}$ for $x<x_{\star}$ and to $k_{2}$ for $x>x_{\star}$, with $F=F_{c}$. 
change branches at some intermediate radius. This selects the flux $F=F_{c}$ as the only physical value and the solution $k_{c}(x)$ that switches from $k_{1}$ to $k_{2}$, as shown by the solid line in Fig. 3. This is similar to the hydrodynamical case $[98,99]$, which selects the only value of the flux that provides a transonic solution that connects the subsonic (i.e., low-velocity) branch at large radii with the supersonic (i.e., high-velocity) branch at low radii.

\section{Boundary conditions}

To obtain the boundary condition at a large radius for the modulus $k(x)$, we consider the behavior of $F(k, x)$ at small $k$. Indeed, as seen in Fig. 1, at large radii the upper boundary $k_{+}(x)$ becomes much smaller than unity. Then, Eq. (88) gives at leading order

$$
k \ll 1: \frac{F}{F_{s}}=x^{2} h k^{2} \sqrt{1-\frac{f\left(1+3 k^{2} / 2\right)}{(1+\alpha)^{2}}} .
$$

At large radii inside the soliton core, we obtain

$$
r \gg r_{\mathrm{sg}}, \quad k \ll 1: \frac{F}{F_{s}}=x^{2} k^{2} \sqrt{\frac{3}{2}\left(\frac{\lambda_{4} \rho}{m^{4}}-k^{2}\right)}
$$

at lowest order in $k^{2}, \Phi$, and $\alpha$, and we used Eqs. (66) and (69). Since at these radii $\lambda_{4} \rho / m^{4} \ll 1$, the small- $k$ expansion is valid up to $k_{+}$, which is thus given by

$$
r \gg r_{\text {sg }}: k_{+}=\sqrt{\frac{\lambda_{4} \rho}{m^{4}}} \ll 1 .
$$

We can see from Eq. (80) that the large-radius boundary condition is, in fact, $k(r)=k_{+}$, when we neglect the velocity as in the analysis of Sec. IV B 1 b. In agreement with Eq. (97), we find that this boundary condition with a zero velocity implies a zero flux $F$. In practice, the matching to the static soliton is not perfect, and there remains a nonzero velocity $\beta^{\prime}$, associated with a nonzero flux $F$. This approximate matching is meaningful as long as the velocity at the outer boundary of the core, $r \sim R_{s} / 10$, is sufficiently small. In other words, it must be much smaller than the free-fall velocity at that radius, and the mass loss onto the $\mathrm{BH}$ should remain much smaller than the soliton mass over the time of interest. We shall check below in Sec. V that this is indeed the case. This also means that at large radii the modulus $k(x)$ must be on the upper branch $k_{2}(x)$, close to the upper boundary $k_{+}(x)$ :

$$
x \gg r_{\mathrm{sg}} / r_{s}: k(x)=k_{2}(x) .
$$

At the Schwarzschild radius, $x \rightarrow 1 / 4, h(x) \rightarrow 16$, and $f(x) \rightarrow 0$. Therefore, the square root in Eq. (88) goes to unity (unless $k \rightarrow 1 / \sqrt{2}$ ). More physically, the square root comes from the velocity factor $\beta^{\prime}$ of Eq. (87), through Eq. (62). Close to the Schwarzschild radius, the velocity should be large and close to unity, as found in Eq. (81), and the self-interaction becomes negligible as we recover the free fall onto the $\mathrm{BH}$. This means that the square root in Eq. (88) goes to unity. Then, the small value of the flux $F$ as compared with the local peak value $F_{\max }(x)$ is reached by having a small value of $k$, thanks to the prefactor $k^{2}$, rather than by having a large value of $k$ close to the upper boundary $k_{+}$where the square root vanishes. This means that at small radii the modulus $k(x)$ must be on the lower branch $k_{1}(x)$, close to zero:

$$
x \simeq 1 / 4: k(x)=k_{1}(x) .
$$

Thus, as announced above, the boundary conditions (99) and (100) imply that the physical solution $k(x)$ must change from the upper to the lower branch, as we get closer to the $\mathrm{BH}$. As explained in Sec. IV D 1, this selects the unique value $F_{c}$ for the flux and a unique function $k(x)$.

\section{Critical solution}

Thus, the unique function $k_{c}(x)$, shown by the solid line in Fig. 3, verifies

$$
\begin{array}{lll}
F=F_{c}, & k_{c}(x)=k_{1}(x) & \text { for } x<x_{\star}, \\
& k_{c}(x)=k_{2}(x) & \text { for } x>x_{\star} .
\end{array}
$$

At the Schwarzschild radius, we obtain

$$
r=r_{s} / 4: k_{c}(1 / 4) \equiv k_{s} \simeq 0.54,
$$

while $k_{c}(x)$ decreases at a large radius. From Eq. (97), with $F=F_{c}$, we obtain at large radii

$$
r \gg r_{\mathrm{sg}}: k_{c}(x)^{2}=\frac{\lambda_{4} \rho}{m^{4}}-\frac{2}{3 x^{4}}\left(\frac{F_{\star} m^{4}}{\lambda_{4} \rho}\right)^{2} .
$$

Equation (62) gives

$$
v_{r} \equiv \frac{\pi \beta^{\prime}}{2 m}=-\sqrt{\frac{h}{f}} \sqrt{(1+\alpha)^{2}-\frac{\pi^{2} f}{\left(1-2 k^{2}\right) 4 \mathbf{K}^{2}}},
$$

where we made the identification $v_{r}=\pi \beta^{\prime} / 2 m$, which holds in the weak-gravity nonrelativistic limit, as explained below Eq. (63). The density $\rho_{\phi}$ defined in Eq. (84) reads

$$
\begin{aligned}
\frac{\left\langle\rho_{\phi}\right\rangle}{\rho_{a}}= & \frac{3 k^{2}}{4\left(1-2 k^{2}\right)}\left[\frac{1-k^{2}}{1-2 k^{2}}+\frac{8 \mathbf{K}^{2} v_{r}^{2}}{\pi^{2} h}\left[1-k^{2}\right.\right. \\
& \left.\left.+\left(2 k^{2}-1\right) C_{2}-k^{2} C_{4}\right]\right],
\end{aligned}
$$

where we took the average over the fast oscillation period and the characteristic density $\rho_{a}$ was defined in Eq. (66). 
Because the metric function $f(r)$ goes to zero at the Schwarzschild radius, as $f(r) \sim\left(r-r_{s} / 4\right)^{2}$, the velocity $v_{r} \sim\left(r-r_{s} / 4\right)^{-1}$ and the density $\langle\rho\rangle \sim\left(r-r_{s} / 4\right)^{-2}$ diverge at the Schwarzschild radius. On the other hand, at a large distance, Eq. (104) gives

$$
r \gg r_{\mathrm{sg}}: v_{r}=-\frac{F_{\star} m^{4}}{\lambda_{4} \rho x^{2}} .
$$

\section{E. Behavior at the Schwarzschild radius in isotropic coordinates}

As for the case of the free scalar field studied in Sec. III, the radial velocity $v_{r}(104)$ and the density $\rho_{\phi}$ (105), defined by the energy-momentum tensor associated with the isotropic metric, diverge at the Schwarzschild radius because of the metric factor $1 / f$. Thus, from Eq. (104) we obtain close to the Schwarzschild radius

$$
\begin{aligned}
r & \rightarrow r_{s} / 4: \frac{\pi \beta^{\prime}}{2 m} \sim-\frac{16(1+\alpha) m r_{s}}{\pi\left(4 r-r_{s}\right)}, \\
\beta & \sim-\frac{4(1+\alpha) m r_{s}}{\pi} \ln \left(\frac{4 r-r_{s}}{4 r_{s}}\right) .
\end{aligned}
$$

\section{F. Behavior at the Schwarzschild radius in Eddington coordinates}

Again, the divergence at the horizon is an artifact due to the choice of coordinates, and by going to the more appropriate Eddington metric (45) we obtain finite quantities. Thus, substituting the result (108) into Eq. (55) and using the Eddington coordinates as in the metric (45), we obtain

$$
\tilde{r} \rightarrow r_{s}: \phi=\phi_{s} \operatorname{cn}\left[\frac{2 \mathbf{K}_{s}}{\pi}(1+\alpha) m(\tilde{t}+\tilde{r}), k_{s}\right],
$$

where the modulus $k_{s}$ at the Schwarzschild radius was obtained in Eq. (102) and the amplitude $\phi_{s}$ is given by Eq. (63) in terms of $k_{s}$. As for the free scalar (48), the scalar field is well defined at the horizon, and we recover an ingoing solution with unity velocity. However, the selfinteractions remain relevant down to the horizon, as (109) differs from the cosine (i.e., harmonic) expression (48) of the free case. We now obtain a nonlinear radial wave, with higher-order harmonics as given by the expansion (57).

\section{G. Density profile}

From Eqs. (46) and (55), using Eqs. (58) and (59), the energy density associated with the Eddington coordinates is given, at leading order in the large- $m$ limit, by

$$
\begin{aligned}
\tilde{\rho}_{\phi}= & \frac{m^{4}}{\lambda_{4}} \frac{k^{2}}{1-2 k^{2}}\left\{\left[1-k^{2}+\left(2 k^{2}-1\right) \mathrm{cn}^{2}-k^{2} \mathrm{cn}^{4}\right]\right. \\
& \times\left[2-f+\frac{1}{f}\left(1-f-\sqrt{1-\frac{\pi^{2} f}{\left(1-2 k^{2}\right) 4 \mathbf{K}^{2}(1+\alpha)^{2}}}\right)^{2}\right] \\
& \left.\times\left(\frac{2 \mathbf{K}(1+\alpha)}{\pi}\right)^{2}+\mathrm{cn}^{2}+\frac{k^{2}}{1-2 k^{2}} \mathrm{cn}^{4}\right\} .
\end{aligned}
$$

This is the generalization of Eq. (49) to the case of quartic self-interaction. In terms of the flux $F_{c}$, we obtain, using Eq. (90) and averaging over the fast oscillations,

$$
\begin{aligned}
\left\langle\tilde{\rho}_{\phi}\right\rangle= & -\frac{F_{c}}{F_{\star} r_{s}^{2}} \frac{k^{2}}{1-2 k^{2}}\left\{\left[1-k^{2}+\left(2 k^{2}-1\right) C_{2}-k^{2} C_{4}\right]\right. \\
& \times\left[2-f+\frac{1}{f}\left(1-f-\sqrt{1-\frac{\pi^{2} f}{\left(1-2 k^{2}\right) 4 \mathbf{K}^{2}(1+\alpha)^{2}}}\right)^{2}\right] \\
& \left.\times\left(\frac{2 \mathbf{K}}{\pi}\right)^{2}+\frac{1}{(1+\alpha)^{2}}\left(C_{2}+\frac{k^{2}}{1-2 k^{2}} C_{4}\right)\right\},
\end{aligned}
$$

which generalizes Eq. (50). Again, this energy density remains finite at the Schwarzschild radius. Neglecting $\alpha \ll 1$ and using $k_{s} \simeq 0.54$, we obtain

$$
\tilde{r}=r_{s}, \quad r=\frac{r_{s}}{4}:\left\langle\tilde{\rho}_{\phi}\right\rangle \simeq 1.2 \frac{m^{4}}{\lambda_{4}} \simeq 0.9 \rho_{a} .
$$

Contrary to the case of the free scalar, the flux $F_{c}$ and the density $\tilde{\rho}_{\phi}$ cannot grow arbitrarily large and take only one specific value, determined by the self-interactions. As could be expected, the density (112) is set by the characteristic density $\rho_{a}$ defined in Eq. (66), which measures the strength of the self-interactions. The unboundedness of the free case is recovered by the fact that $\left\langle\tilde{\rho}_{\phi}\right\rangle \rightarrow \infty$ when $\lambda_{4} \rightarrow 0$.

We can see that all terms in Eq. (110) are of the same order. This means that the terms associated with the selfinteraction potential are of the same order as those associated with the quadratic part. Thus, close to the $\mathrm{BH}$, the self-interaction potential can no longer be treated as a perturbation, which was the case on cosmological and galactic scales. This also corresponds to the fact that the modulus $k_{c}$ becomes of the order of unity close to the Schwarzschild radius [see (102)], and the Jacobi elliptic function significantly deviates from a cosine. Moreover, we can see that the slope of the density profile is different from the exponent $-3 / 2$ obtained in the free case in Eq. (52). Indeed, from Eq. (96), we obtain

$$
r_{s} \ll r \ll r_{\mathrm{sg}}: k \simeq k_{+} \simeq \sqrt{\frac{2 r_{s}}{3 r}} .
$$

This leads to 


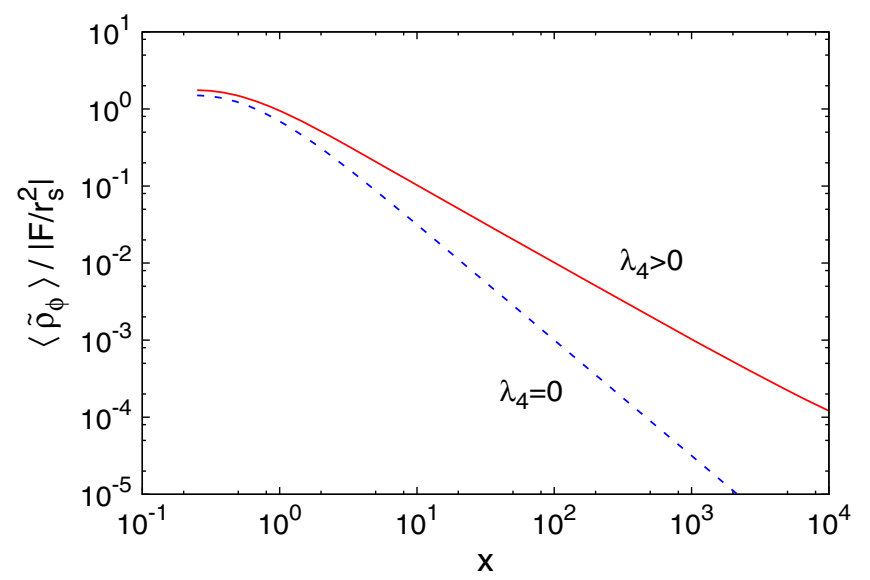

FIG. 4. Scalar-field energy density computed in the Eddington metric, from the Schwarzschild radius up to $10^{4} r_{s}$, where the metric potentials are still dominated by the central $\mathrm{BH}$. We show the free case (50) (dashed line) and the self-interaction case (111) (solid line), for the same value $F_{c}$ of the flux.

$$
r_{s} \ll r \ll r_{\mathrm{sg}}:\left\langle\tilde{\rho}_{\phi}\right\rangle \propto r^{-1} \text { and } \quad v_{r} \propto r^{-1} \text {. }
$$

As compared with the free case (52), the density falls off more slowly at large radii while the velocity decreases faster.

We show in Fig. 4 the scalar-field profiles of the free and interacting cases, for the same value $F_{c}$ of the flux. Both densities are of the same order at the Schwarzschild radius, but we can clearly see the two different slopes for $r \gg r_{s}$, with the slower falloff for the interacting case. This corresponds in turns to a faster decay of the radial velocity. This is not surprising, since the pressure support provided by the self-interaction balances gravity and stabilizes the scalar-field soliton obtained at large radii, as recalled in Sec. IV B 1, and slows down the infall onto the central BH at smaller radii. On the other hand, near the Schwarzschild radius, gravity cannot be resisted, and the radial velocity becomes of the order of unity in both cases.

\section{H. Transition radius}

From Eq. (114), we obtain the more explicit scalings

$$
r_{s}<r<r_{\mathrm{sg}}: \tilde{\rho}_{\phi} \sim \rho_{a} \frac{r_{s}}{r}, \quad v_{r} \sim-\frac{r_{s}}{r} .
$$

This BH-dominated regime stops at the radius $r_{\text {sg }}$ where the scalar-field density has decreased down to the soliton core density $\rho_{s}$. This gives

$$
r_{\mathrm{sg}}=r_{s} \frac{\rho_{a}}{\rho_{s}}
$$

From Eqs. (11) and (66), we find at this radius

$$
r=r_{\mathrm{sg}}: \Phi_{\mathrm{BH}}=-\frac{\rho_{s}}{2 \rho_{a}}, \quad \Phi_{\mathrm{I}}=\frac{\rho_{s}}{\rho_{a}}, \quad v_{r} \sim-\frac{\rho_{s}}{\rho_{a}},
$$

where $\Phi_{\mathrm{BH}}$ is the Newtonian potential associated with the central BH. Normalizing the scalar-field Newtonian potential $\Phi_{\phi}$ at large radii, beyond the soliton radius, it follows the soliton profile (69) down to $r_{\mathrm{sg}}$, where the mass distribution starts to deviate from the flat soliton solution. Thus, we also have

$$
r=r_{\mathrm{sg}}: \Phi_{\phi}=\alpha-\Phi_{\mathrm{I}} \sim-\frac{\rho_{s}}{\rho_{a}}
$$

Then, we can check that we indeed have $\Phi_{\phi} \sim \Phi_{\mathrm{BH}}$ at the transition radius $r_{\text {sg }}$ given by Eq. (116). From Eq. (106), we find that at larger radii, up to the soliton radius $R_{s}$, we have

$$
r_{\mathrm{sg}}<r<R_{s}: \tilde{\rho}_{\phi} \sim \rho_{s}, \quad v_{r} \sim-\frac{\rho_{s}}{\rho_{a}} \frac{r_{\mathrm{sg}}^{2}}{r^{2}} .
$$

Of course, the spherical flux $r^{2} \tilde{\rho}_{\phi} v_{r}$ scales as $r^{0}$, that is, remains constant, in both small and large radii regimes (115) and (119).

For this analysis to be valid, we must check that the transition radius $r_{\mathrm{sg}}$ is smaller than the soliton radius $R_{s}$. Using Eqs. (68) and (116), with $R_{s} \sim r_{a}$, we find that $r_{\text {sg }}<$ $R_{s}$ corresponds to $M<M_{s}$, where $M_{s} \sim \rho_{s} r_{a}^{3}$ is the soliton mass. The ratio $M / M_{h}$ of the supermassive central $\mathrm{BH}$ mass to the halo dark matter mass is of the order of $10^{-5}-$ $10^{-4}$ [100]. On the other hand, the ratio $M_{s} / M_{h}$ of the soliton mass to the halo dark matter mass is of the order of $10^{-3}-1$ [89]. Therefore, we typically have $M \ll M_{s}$, and the radius $r_{\mathrm{sg}}$ that marks the central region dominated by the $\mathrm{BH}$ gravitational potential is significantly smaller than the soliton radius $R_{s}$.

\section{Scalar dark matter mass at small radii}

Some scalar-field dark matter models can be constrained by the measurement of stellar dynamics at small radii, near the central supermassive BH. For instance, an extended dark matter distribution around the $\mathrm{BH}$ can affect the orbits of local stars and lead to significant precession. This requires accurate measurements at very small radii, which start to be available for a few cases, such as the Sgr A* BH in the Milky Way or the M87* BH in the M87 galaxy. In the first case, the mass distribution is known up to the few percent level [90], whereas for the latter one, the distribution is constrained at the order of $10 \%$ [91]. This type of observation has been recently studied in this context $[71,72,101]$.

In our case, where the scalar dark matter is supported by the self-interaction pressure, the orders of magnitude are significantly different from the fuzzy dark matter scenario. Let us consider the case $\rho_{a} \sim 1 \mathrm{eV}^{4}$ and $R_{s} \simeq 20 \mathrm{kpc}$. For the Milky Way, with a dark matter halo mass $M_{h} \sim 10^{12} M_{\odot}$ and a soliton mass ratio $M_{s} / M_{h} \sim 0.03$ [89], we obtain a scalar soliton mass $M_{s} \simeq 3 \times 10^{10} M_{\odot}$. On the other hand, 
the central supermassive $\mathrm{BH}$ has a mass $M \simeq 4.3 \times 10^{6} M_{\odot}$. This gives a Schwarzschild radius $r_{s} \simeq 4 \times 10^{-7} \mathrm{pc}$ and a transition radius $r_{\mathrm{sg}} \simeq 0.1 \mathrm{pc}$. From Eq. (119), we have in the large-radius regime $r_{\mathrm{sg}}<r<R_{s}$ the scaling $M_{\phi}(<r) \propto r^{3}$. Therefore, we obtain at the transition radius $M_{\phi}(<0.1 \mathrm{pc}) \simeq 4 \times 10^{-6} M_{\odot}$. From Eq. (115), we have in the small-radius regime $r_{s}<r<r_{\mathrm{sg}}$ the scaling $M_{\phi}(<r) \propto r^{2}$. This gives, in particular, $M_{\phi}(<0.005 \mathrm{pc}) \simeq$ $10^{-8} M_{\odot}$. The observational constraints are $M_{\phi}<10^{5} M_{\odot}$ within $0.005 \mathrm{pc}$ and $M_{\phi}<10^{6} M_{\odot}$ within $0.3 \mathrm{pc}$. Thus, the soliton mass at small radii is far below the observational upper bounds. On the other hand, these measurements could constrain scalar-field models such as the one studied in this paper but with very different parameters, which would then play no role on galactic scales and only become relevant at milliparsec scales.

\section{LIFETIME OF THE SCALAR-FIELD SOLITON}

At the typical soliton radius $r_{a}=R_{s} / \pi$, Eqs. (116) and (119) give for the radial velocity $v_{r}$ and the evolution timescale $t_{c}$, respectively,

$$
v_{r}\left(r_{a}\right) \sim-\frac{\rho_{a}}{\rho_{s}} \frac{r_{s}^{2}}{r_{a}^{2}}, \quad t_{c} \equiv \frac{r_{a}}{\left|v_{r}\right|} \sim r_{a} \frac{\rho_{s}}{\rho_{a}} \frac{r_{a}^{2}}{r_{s}^{2}} .
$$

To compare the time $t_{c}$ with cosmological timescales, we define the Hubble time $t_{H}$ and Hubble radius $R_{H}$ as, respectively,

$$
t_{H}=1 / H, \quad R_{H}=1 / H,
$$

and we obtain

$$
t_{c} \sim t_{H}\left(\frac{\bar{\rho}_{c}}{\rho_{a}}\right)^{5 / 2} \frac{\rho_{s}}{\bar{\rho}_{c}}\left(\frac{R_{H}}{r_{s}}\right)^{2},
$$

where $\bar{\rho}_{c}=3 H^{2} /(8 \pi \mathcal{G})$ is the cosmological critical density. This also reads at $z=0$ as

$$
t_{c} \sim 10^{3} t_{H} \frac{\rho_{s}}{\bar{\rho}_{c}}\left(\frac{\rho_{a}}{1 \mathrm{eV}^{4}}\right)^{-5 / 2}\left(\frac{M}{10^{8} M_{\odot}}\right)^{-2} .
$$

For the soliton to have a radius of $20 \mathrm{kpc}$, so that it shows a significant departure from the CDM profiles on galactic scales, we must have $\rho_{a} \sim 1 \mathrm{eV}^{4}$ [89]. Larger characteristic densities lead to smaller soliton radii. We typically have $\rho_{s} / \bar{\rho}_{c} \sim 10^{5}$ for the DM overdensity in the soliton core. Therefore, we find that $t_{c} \gg t_{H}$. This means that the DM solitonic cores can easily survive until today, despite the infall of their inner layers onto the central supermassive $\mathrm{BH}$.

We also find that astrophysical stellar mass BHs cannot eat a significant fraction of the galactic DM soliton. Indeed, for $N$ BHs of unit solar mass, the typical timescale for the soliton depletion reads

$$
t_{N} \sim 10^{19} \frac{t_{H}}{N} \frac{\rho_{s}}{\bar{\rho}_{c}}\left(\frac{\rho_{a}}{1 \mathrm{eV}^{4}}\right)^{-5 / 2} .
$$

Since we typically have $N<10^{11}$, as only a fraction of the galactic baryonic mass can be within stellar BHs, we obtain $t_{N} \gg 10^{8} t_{H}$ and the soliton mass loss is negligible.

\section{DISCUSSION AND CONCLUSION}

In this work, we have analyzed steady solutions of coherent scalar fields in galactic centers that harbor a supermassive central BH. Neglecting the central BH, such ultralight scalar DM typically builds a stationary coherent profile, called a soliton, with a finite radius $R_{s}$ and a flat core. This soliton is also embedded in an extended halo of fluctuating density granules, with a spherically averaged density profile that is similar to the NFW profile [87] found in numerical simulations of standard collisionless dark matter. If $R_{s}$ is of the order of a few kiloparsecs, this flattened dark matter profile can have interesting observational consequences for cosmological and galactic studies. In contrast with the fuzzy dark matter scenarios, with a scalar mass $m \sim 10^{-22} \mathrm{eV}$, where the soliton is due to the balance between gravity and the quantum pressure (associated with the wave features of the scalar field), we focus on the case of large scalar mass, typically $m \gg 10^{-18} \mathrm{eV}$, where gravity is instead counterbalanced by the repulsive self-interaction associated with a quartic potential and the quantum pressure is negligible.

In this paper, we have considered the impact of the central supermassive BH on the profile of this soliton and its lifetime, as it gradually falls onto the $\mathrm{BH}$. As we focus on the limit of large scalar mass, we are able to perform a fully nonrelativistic study, from the radius $R_{s}$ of the soliton down to the Schwarzschild radius $r_{s}$. For simplicity, we discard baryonic effects, but the main features of both the relativistic infall at small radii and the soliton core at large radii should remain valid. Baryonic matter will increase only somewhat the soliton density at intermediate radii, where it dominates over both the central $\mathrm{BH}$ and scalar gravitational fields. Then, our analysis extends from the large-radius regime $r \lesssim R_{s}$ dominated by the scalar dark matter self-gravity down to the small-radius regime $r \sim r_{s}$ dominated by the $\mathrm{BH}$ gravity. The boundary conditions at both ends determine the profile and the steady infall onto the supermassive central $\mathrm{BH}$.

First, we have studied the free massive case, associated with a quadratic scalar potential. As the scalar-field equation of motion is linear, this behaves in a fashion similar to a collection of independent particles, with a flux onto the central BH that is arbitrary and unbounded, proportional to the density at large radii. As expected, at 
the Schwarzschild radius, the scalar field takes the form of a harmonic ingoing wave.

Then, we have extended the analysis to the self-interacting case defined by a repulsive quartic interaction. The limit of large scalar mass allows us to perform a fully nonlinear study, at all orders in the coupling constant. These nonlinear dynamics generate harmonics of all orders. The effective pressure associated with the repulsive quartic interaction slows down the infall onto the central BH. In a fashion similar to the hydrodynamical case of polytropic fluids, general relativity actually selects a unique critical value $F_{c}$ for the flux of the steady infall onto the $\mathrm{BH}$. This is similar to the transonic solution of the hydrodynamical case, with a continuous switch from a low-velocity branch at large radii, which converges to the soliton solution with a negligible radial velocity, to a high-velocity branch at small radii, with a radial velocity that becomes relativistic. At the Schwarzschild radius, the scalar field takes again the form of an ingoing wave with unit velocity, as the self-interaction pressure cannot resist the $\mathrm{BH}$ gravity, but it is now a nonlinear wave that contains harmonics of all orders. We find that in the central region, dominated by the $\mathrm{BH}$ gravity, the scalar density profile and the radial velocity decay as $1 / r$. Beyond a transition radius $r_{\mathrm{sg}}$, the scalar self-gravity becomes dominant, and the scalar density follows the flat core $\rho_{s}$ of the soliton, while the negligible radial velocity decays as $1 / r^{2}$.

The critical flux $F_{c}$ gives a lifetime $t_{c}$ of the soliton that is much longer than the age of the Universe. This implies that the soliton solutions generated by this scalar DM scenario are not destroyed by the supermassive central BH and are relevant. However, because of the large soliton radius $R_{s}$ (as we focus on models that could have some impact on galactic scales), the scalar dark matter mass at small radii is very small and much below the observational upper bounds provided by stellar dynamics close to the central supermassive $\mathrm{BH}$.

By increasing the scalar mass or decreasing the quartic coupling constant, the core density of the soliton becomes greater while its radius diminishes. This would, in turn, increase the scalar density near the black hole and lead to stronger effects on the stellar dynamics in this central region. Better constraints on the mass of scalar dark matter in this regime would certainly require one to calibrate the models from large to small scales using dedicated numerical simulations, in particular, to estimate the expected mass of such small solitons. More generally, simulations of selfinteracting scalar dark matter would help understand the complex scalar dynamics from cosmological scales, outside of coherent solitons, down to small subgalactic scales, which involve soliton collisions and possible relaxation processes that are difficult to predict in nonlinear regimes.

\section{ACKNOWLEDGMENTS}

This work is supported in part by the EU Horizon 2020 research and innovation program under the MarieSklodowska Grant No. 690575. This article is based upon work related to the COST Action CA15117 (CANTATA) supported by COST (European Cooperation in Science and Technology). The work by J. A. R. C. is partially supported by the MINECO (Spain) Project No. FIS2016-78859-P (AEI/FEDER, UE). This work was made possible by Institut Pascal at Université Paris-Saclay with the support of the P2I and SPU research departments and the P2IO Laboratory of Excellence (program Investissements davenir ANR-11-IDEX-0003-01 Paris-Saclay and ANR-10LABX-0038), as well as the IPhT.
[1] R. D. Peccei and H. R. Quinn, Phys. Rev. Lett. 38, 1440 (1977).

[2] F. Wilczek, Phys. Rev. Lett. 40, 279 (1978).

[3] S. Weinberg, Phys. Rev. Lett. 40, 223 (1978).

[4] D. J. E. Marsh, Phys. Rep. 643, 1 (2016).

[5] L. Hui, J. P. Ostriker, S. Tremaine, and E. Witten, Phys. Rev. D 95, 043541 (2017).

[6] A. Sarkar, R. Mondal, S. Das, S. Sethi, S. Bharadwaj, and D. J. E. Marsh, J. Cosmol. Astropart. Phys. 04 (2016) 012.

[7] T. Kobayashi, R. Murgia, A. De Simone, V. Iršič, and M. Viel, Phys. Rev. D 96, 123514 (2017).

[8] C. Abel et al., Phys. Rev. X 7, 041034 (2017).

[9] N. Banik, A. J. Christopherson, P. Sikivie, and E. M. Todarello, Phys. Rev. D 95, 043542 (2017).

[10] S. Hirano, J. M. Sullivan, and V. Bromm, Mon. Not. R. Astron. Soc. 473, L6 (2018).
[11] J. P. Conlon, F. Day, N. Jennings, S. Krippendorf, and F. Muia, Mon. Not. R. Astron. Soc. 473, 4932 (2018).

[12] R. Brito, S. Ghosh, E. Barausse, E. Berti, V. Cardoso, I. Dvorkin, A. Klein, and P. Pani, Phys. Rev. Lett. 119, 131101 (2017).

[13] R. Brito, S. Ghosh, E. Barausse, E. Berti, V. Cardoso, I. Dvorkin, A. Klein, and P. Pani, Phys. Rev. D 96, 064050 (2017).

[14] A. Sarkar, S. K. Sethi, and S. Das, J. Cosmol. Astropart. Phys. 07 (2017) 012.

[15] J. A. D. Diacoumis and Y. Y. Y. Wong, J. Cosmol. Astropart. Phys. 09 (2017) 011.

[16] W. Hu, R. Barkana, and A. Gruzinov, Phys. Rev. Lett. 85, 1158 (2000).

[17] M. S. Turner, Phys. Rev. D 28, 1243 (1983). 
[18] M. C. Johnson and M. Kamionkowski, Phys. Rev. D 78, 063010 (2008).

[19] J. A. R. Cembranos, C. Hallabrin, A. L. Maroto, and S. J. N. Jareño, Phys. Rev. D 86, 021301 (2012).

[20] J. A. R. Cembranos, A. L. Maroto, and S. J. Núñez Jareño, Phys. Rev. D 87, 043523 (2013).

[21] J. A. R. Cembranos, A. L. Maroto, and S. J. Núñez Jareño, J. Cosmol. Astropart. Phys. 03 (2014) 042.

[22] C. Álvarez-Luna and J. A. R. Cembranos, J. High Energy Phys. 07 (2019) 110.

[23] J.-c. Hwang and H. Noh, Phys. Lett. B 680, 1 (2009).

[24] C.-G. Park, J.-c. Hwang, and H. Noh, Phys. Rev. D 86, 083535 (2012).

[25] R. Hlozek, D. Grin, D. J. E. Marsh, and P. G. Ferreira, Phys. Rev. D 91, 103512 (2015).

[26] J. A. R. Cembranos, A. L. Maroto, and S. J. Núñez Jareño, J. High Energy Phys. 03 (2016) 013.

[27] J. A. R. Cembranos, A. L. Maroto, and S. J. Núñez Jareño, J. High Energy Phys. 02 (2017) 064.

[28] H.-Y. Schive, T. Chiueh, and T. Broadhurst, Nat. Phys. 10, 496 (2014).

[29] T. Broadhurst, H. N. Luu, and S. H. H. Tye, arXiv:1811. 03771.

[30] J. P. Ostriker and P. J. Steinhardt, Science 300, 1909 (2003).

[31] J. A. R. Cembranos, J.L. Feng, A. Rajaraman, and F. Takayama, Phys. Rev. Lett. 95, 181301 (2005).

[32] D. H. Weinberg, J. S. Bullock, F. Governato, R. Kuzio de Naray, and A. H. G. Peter, Proc. Natl. Acad. Sci. U.S.A. 112, 12249 (2015).

[33] A. Pontzen and F. Governato, Nature (London) 506, 171 (2014).

[34] M. Boylan-Kolchin, J. S. Bullock, and M. Kaplinghat, Mon. Not. R. Astron. Soc. 415, L40 (2011).

[35] B. Moore, S. Ghigna, F. Governato, G. Lake, T. R. Quinn, J. Stadel, and P. Tozzi, Astrophys. J. 524, L19 (1999).

[36] W. J. G. de Blok, Adv. Astron. 2010, 789293 (2010).

[37] J. A. R. Cembranos, A. L. Maroto, S. J. Núñez Jareño, and H. Villarrubia-Rojo, J. High Energy Phys. 08 (2018) 073.

[38] A. Arbey, J. Lesgourgues, and P. Salati, Phys. Rev. D 64, 123528 (2001).

[39] J. Lesgourgues, A. Arbey, and P. Salati, New Astron. Rev. 46, 791 (2002).

[40] P.-H. Chavanis, Phys. Rev. D 84, 043531 (2011).

[41] P. H. Chavanis and L. Delfini, Phys. Rev. D 84, 043532 (2011).

[42] H.-Y. Schive, M.-H. Liao, T.-P. Woo, S.-K. Wong, T. Chiueh, T. Broadhurst, and W. Y. P. Hwang, Phys. Rev. Lett. 113, 261302 (2014).

[43] D. J. E. Marsh and A.-R. Pop, Mon. Not. R. Astron. Soc. 451, 2479 (2015)

[44] E. Calabrese and D. N. Spergel, Mon. Not. R. Astron. Soc. 460, 4397 (2016).

[45] S.-R. Chen, H.-Y. Schive, and T. Chiueh, Mon. Not. R. Astron. Soc. 468, 1338 (2017).

[46] B. Schwabe, J. C. Niemeyer, and J. F. Engels, Phys. Rev. D 94, 043513 (2016).

[47] J. Veltmaat and J. C. Niemeyer, Phys. Rev. D 94, 123523 (2016).
[48] A. X. González-Morales, D. J. E. Marsh, J. Peñarrubia, and L. A. Ureña-López, Mon. Not. R. Astron. Soc. 472, 1346 (2017).

[49] V. H. Robles and T. Matos, Mon. Not. R. Astron. Soc. 422, 282 (2012).

[50] T. Bernal, L. M. Fernández-Hernández, T. Matos, and M. A. Rodríguez-Meza, Mon. Not. R. Astron. Soc. 475, 1447 (2018).

[51] P. Mocz, M. Vogelsberger, V. H. Robles, J. Zavala, M. Boylan-Kolchin, A. Fialkov, and L. Hernquist, Mon. Not. R. Astron. Soc. 471, 4559 (2017).

[52] K. Mukaida, M. Takimoto, and M. Yamada, J. High Energy Phys. 03 (2017) 122.

[53] J. Vicens, J. Salvado, and J. Miralda-Escudé, arXiv:1802. 10513.

[54] N. Bar, D. Blas, K. Blum, and S. Sibiryakov, Phys. Rev. D 98, 083027 (2018).

[55] J. Eby, K. Mukaida, M. Takimoto, L. C. R. Wijewardhana, and M. Yamada, Phys. Rev. D 99, 123503 (2019).

[56] B. Bar-Or, J.-B. Fouvry, and S. Tremaine, Astrophys. J. 871, 28 (2019).

[57] D. J. E. Marsh and J. C. Niemeyer, Phys. Rev. Lett. 123, 051103 (2019).

[58] P.-H. Chavanis, Phys. Rev. D 100, 083022 (2019).

[59] R. Emami, T. Broadhurst, G. Smoot, T. Chiueh, and H. N. Luu, arXiv:1806.04518.

[60] D. G. Levkov, A. G. Panin, and I. I. Tkachev, Phys. Rev. Lett. 121, 151301 (2018).

[61] T. Broadhurst, I. de Martino, H. N. Luu, G. F. Smoot, and S. H. H. Tye, arXiv:1902.10488.

[62] K. Hayashi and I. Obata, Mon. Not. R. Astron. Soc. 491, 615 (2020).

[63] N. Bar, K. Blum, J. Eby, and R. Sato, Phys. Rev. D 99, 103020 (2019).

[64] J. Kormendy and D. Richstone, Annu. Rev. Astron. Astrophys. 33, 581 (1995).

[65] L. Ferrarese and H. Ford, Space Sci. Rev. 116, 523 (2005).

[66] R. Narayan, New J. Phys. 7, 199 (2005).

[67] M. C. Ferreira, C. F. B. Macedo, and V. Cardoso, Phys. Rev. D 96, 083017 (2017).

[68] M. Bošković, F. Duque, M. C. Ferreira, F. S. Miguel, and V. Cardoso, Phys. Rev. D 98, 024037 (2018).

[69] V. Cardoso, Ó. J. C. Dias, G. S. Hartnett, M. Middleton, P. Pani, and J. E. Santos, J. Cosmol. Astropart. Phys. 03 (2018) 043.

[70] H. Davoudiasl and P. B. Denton, Phys. Rev. Lett. 123, 021102 (2019).

[71] V. Desjacques and A. Nusser, Mon. Not. R. Astron. Soc. 488, 4497 (2019).

[72] N. Bar, K. Blum, T. Lacroix, and P. Panci, J. Cosmol. Astropart. Phys. 07 (2019) 045.

[73] L. Hui, D. Kabat, X. Li, L. Santoni, and S. S. C. Wong, J. Cosmol. Astropart. Phys. 06 (2019) 038.

[74] K. Clough, P. G. Ferreira, and M. Lagos, Phys. Rev. D 100, 063014 (2019).

[75] M. Khlopov, B. A. Malomed, and I. B. Zeldovich, Mon. Not. R. Astron. Soc. 215, 575 (1985).

[76] J. Goodman, New Astron. 5, 103 (2000).

[77] B. Li, T. Rindler-Daller, and P. R. Shapiro, Phys. Rev. D 89, 083536 (2014). 
[78] A. Suárez and P.-H. Chavanis, Phys. Rev. D 95, 063515 (2017).

[79] A. Suárez and P.-H. Chavanis, Phys. Rev. D 92, 023510 (2015).

[80] A. Suárez and P.-H. Chavanis, Phys. Rev. D 98, 083529 (2018).

[81] F. X. L. Cedeño, A. X. González-Morales, and L. A. Ureña-López, Phys. Rev. D 96, 061301 (2017).

[82] V. Desjacques, A. Kehagias, and A. Riotto, Phys. Rev. D 97, 023529 (2018).

[83] J. Fan, Phys. Dark Universe 14, 84 (2016).

[84] T. Rindler-Daller and P. R. Shapiro, Mon. Not. R. Astron. Soc. 422, 135 (2012).

[85] P. S. B. Dev, M. Lindner, and S. Ohmer, Phys. Lett. B 773, 219 (2017).

[86] B. Li, P. R. Shapiro, and T. Rindler-Daller, Phys. Rev. D 96, 063505 (2017).

[87] J. F. Navarro, C. S. Frenk, and S. D. M. White, Astrophys. J. 462, 563 (1996).

[88] K. Inayoshi, E. Visbal, and Z. Haiman, arXiv:1911.05791.

[89] P. Brax, J. A. R. Cembranos, and P. Valageas, Phys. Rev. D 100, 023526 (2019).
[90] Q. Yu, F. Zhang, and Y. Lu, Astrophys. J. 827, 114 (2016).

[91] K. Akiyama et al. (Event Horizon Telescope Collaboration), Astrophys. J. 875, L6 (2019).

[92] E. Poisson, A Relativist's Toolkit: The Mathematics of Black-Hole Mechanics (Cambridge University Press, Cambridge, England, 2009).

[93] M. Blau, Lecture Notes on General Relativity, http://www .blau.itp.unibe.ch/GRLecturenotes.html.

[94] E. Madelung, Z. Phys. 40, 322 (1927).

[95] I. Kovacic and M. Brennan, The Duffing Equation: Nonlinear Oscillators and their Behaviour (Wiley, New York, 2011).

[96] I. S. Gradshteyn and I. M. Ryzhik, Table of Integrals, Series, and Products, 4th ed. (Academic Press, New York, 1965), http://openlibrary.org/books/OL5955048M.

[97] P. Byrd and M. Friedman, Handbook of Elliptic Integrals for Engineers and Scientists (Springer, Berlin, 1971).

[98] H. Bondi, Mon. Not. R. Astron. Soc. 112, 195 (1952).

[99] F. C. Michel, Astrophys. Space Sci. 15, 153 (1972).

[100] L. Ferrarese, Astrophys. J. 578, 90 (2002).

[101] E. Y. Davies and P. Mocz, arXiv:1908.04790. 